\title{
Combining set-theoretic UIO and invariant sets for optimal guaranteed robust fault detection and isolation
}

\author{
Feng $\mathrm{Xu}^{\mathrm{a}}$, Junbo Tan ${ }^{\mathrm{d}}$, Ye Wang ${ }^{\mathrm{b}, *}$, Vicenç Puig ${ }^{\mathrm{c}}$, Xueqian Wang ${ }^{\mathrm{a}, *}$ \\ ${ }^{a}$ Center for Artificial Intelligence and Robotics, Graduate School at Shenzhen, Tsinghua University, 518055 Shenzhen, PR China \\ ${ }^{\mathrm{b}}$ College of Automation, Harbin Engineering University, 150001 Harbin, PR China \\ c Institut de Robòtica i Informàtica Industrial (CSIC-UPC), Universitat Politècnica de Catalunya, Llorens i Artigas, 4-6, 08028 Barcelona, Spain \\ ${ }^{\mathrm{d}}$ Navigation and Control Research Center, School of Automation, Tsinghua University, 100084 Beijing, PR China
}

\section{A R T I C L E I N F O}

\section{Article history:}

Received 15 November 2018

Received in revised form 11 March 2019

Accepted 2 April 2019

Available online 3 June 2019

\section{Keywords:}

Guaranteed fault detection and isolation

Optimality

Set-theoretic unknown input observer

Invariant sets

\begin{abstract}
A B S T R A C T
This paper proposes an optimal guaranteed robust fault detection and isolation (FDI) method combing the set-theoretic unknown input observer (SUIO) and the robust positively invariant (RPI) sets for linear time-invariant (LTI) systems. The optimality of the proposed FDI method is achieved under a two-layer framework. The first layer allows to design a single optimal FDI-oriented SUIO with RPI set separationbased guaranteed FDI conditions to reduce the FDI conservatism of single observer. The second layer consists in designing an optimal observer configuration for a bank of optimal SUIOs to reduce the FDI conservatism of all the SUIOs together. Finally, the effectiveness of the proposed guaranteed robust FDI method is illustrated by using a four-tank system.
\end{abstract}

\section{Introduction}

During the past decades, a large number of techniques have been investigated for robust FDI [1,2]. Different types of observers (e.g., Luenberger observer, unknown input observer (UIO) or Kalman filter etc.) have been used to achieve different FDI performances [3-6]. Among these techniques, the UIO has been proven as a useful tool for robust FDI $[7,8,6,9,10]$. The existing works on the UIO can be classified into two categories. In the first category, the design of UIOs is based on the classical matrix rank-based unknown input decoupling condition [11-13]. In the other category, the UIO-based methods seek to reduce the conservatism of the classical unknown input decoupling condition by considering a priori knowledge of system/unknown inputs [14-17]. Further works on the use of UIOs can be found in [18-20]. In order to overcome the conservatism of the classical unknown inputs decoupling condition and simultaneously keep FDI robustness, our previous works [17,21] proposed the set-theoretic unknown input observer by combining the UIO and the set theory for a mixed active and passive decoupling of unknown inputs. The focus of the current paper is to further com-

\footnotetext{
* Corresponding authors.

E-mail addresses: xu.feng@sz.tsinghua.edu.cn (F.Xu), yewang@hrbeu.edu.cn (Y. Wang), wang.xq@sz.tsinghua.edu.cn (X. Wang).
}

bine the SUIO and the invariant sets in order to achieve optimal guaranteed robust FDI.

Regarding robust FDI based on the set theory, there exist three types of methods in the literature, i.e., the interval observer-based methods, the set-membership estimation-based methods and the invariant set-based methods [22-27]. The first two methods implement robust FDI by propagating the bounds of unknown inputs and noises through a system model to generate a residual interval/set at each time instant. FDI decisions are made by checking whether the residuals are inside the corresponding intervals/sets or not $[22,24]$. In the third method, a fixed set is constructed by using invariant sets to describe the system steady-state behaviors and then FDI decisions are made by checking the inclusion between the residuals and the corresponding healthy and faulty sets. Among these robust FDI methods aforementioned, only the invariant set-based methods can provide guaranteed robust FDI, which construct healthy and faulty invariant sets to describe the healthy and faulty system behaviors, respectively. If the healthy and faulty sets are separate from each other, it is guaranteed that the faults can be detected and isolated once they occur [3,23,28,29].

This paper considers guaranteed robust FDI using a bank of observers [28-30]. Different from the existing FDI schemes using a bank of observers, the proposed FDI method here uses invariant sets to establish guaranteed robust FDI conditions based on the separation of healthy and faulty sets, and employs the SUIO to decouple part of unknown inputs and simultaneously keep FDI 
robustness with respect to the other unknown inputs. Different from the existing invariant set-based guaranteed robust FDI methods $[3,23,28,29]$, this proposed method focuses on the optimality of guaranteed robust FDI and reduces the FDI conservatism. In order to reach this objective, the proposed method uses the UIO instead of the Luenberger observer in the existing invariant set-based FDI schemes to remove the effect of some unknown inputs. Moreover, a two-layer framework is proposed to minimize the effect of unknown inputs and noises on guaranteed robust FDI both for the cases of single observer and a bank of observers. Theoretically, this proposed method can obtain less conservative guaranteed robust FDI decisions than the existing guaranteed robust FDI methods. It should be mentioned that the optimality problem of the FDI scheme using a bank of observers is not investigated yet and the main contributions of this paper are summarized as follows:

- a method to establish invariant set separation-based guaranteed FDI conditions for single optimal SUIO is proposed, which reduces the FDI conservatism of single observer but simultaneously obtains guaranteed robust FDI results (see Section 4);

- a method to design an optimal observer configuration for a bank of SUIOs is proposed, which aims to minimize the effect of unknown inputs on guaranteed robust FDI to reduce the conservatism (see Section 5).

This paper is organized in the following. Section 2 presents some preliminaries. Section 3 introduces the system and the SUIO. In Section 4, the optimal FDI-oriented SUIO is designed and the corresponding invariant set separation-based guaranteed FD conditions are established for the optimal FDI-oriented SUIO. In Section 5 , the optimal partitioning of control and unknown inputs is systematically analyzed and an optimal observer configuration is then designed for the objective of optimal guaranteed robust FDI. In Section 6, a case study is used to illustrate the effectiveness of the proposed FDI method. Finally, this paper is concluded in Section 7.

\section{Preliminaries}

Some symbols are defined in the following. The identity matrix and the matrix full of 1 are denoted as $I$ and $\mathbf{I}$, respectively. $\operatorname{diag}(\cdot)$ denotes a diagonal matrix whose diagonal elements are composed of a vector. For a matrix $A,\|A\|_{F}$ denotes its Frobenious norm (i.e., $F$-norm). For matrices $A$ and $B$, and vectors $x$ and $y$, the absolute value symbols $|A|$ and $|x|$ and the inequalities $A \leq B$ and $x \leq y$ should be understood elementwise.

Definition 1. A zonotope $Z$ is defined as $Z=g \oplus H \mathbb{B}^{t}$, where $g$ and $H$ are its center and generator matrix, respectively, $\mathbb{B}^{t}$ is a box composed of $t$ unitary intervals $[-1,1]$ and the symbol $\oplus$ denotes the Minkowski sum.

Property 1. Given two zonotopes $Z_{1}=g_{1} \oplus H_{1} \mathbb{B}^{t_{1}}$ and $Z_{2}=g_{2} \oplus$ $H_{2} \mathbb{B}^{t_{2}}, Z_{1} \oplus Z_{2}=\left\{g_{1}+g_{2}\right\} \oplus\left[\begin{array}{ll}H_{1} & H_{2}\end{array}\right] \mathbb{B}^{t_{1}+t_{2}}$.

Property 2. Given a zonotope $Z=g \oplus H \mathbb{B}^{t}$ and a compatible matrix $K, K Z=K g \oplus K H \mathbb{B}^{t}$.

Property 3. ([31,32]) Given a zonotope $Z=g \oplus H \mathbb{B}^{t} \subset \mathbb{R}^{n}$ and an integer $s$ (with $n<s<t$ ), denote by $\hat{H}$ the matrix resulting from the reordering of the columns of the matrix $H$ in decreasing Euclidean norm, it is obtained that $Z \subseteq g \oplus\left[\begin{array}{ll}\hat{H}_{T} & Q\end{array}\right] \mathbb{B}^{s}$ where $\hat{H}_{T}$ is obtained from the first $s-n$ columns of the matrix $\hat{H}$ and $Q \in \mathbb{R}^{n \times n}$ is a diagonal matrix whose elements satisfy $Q_{i i}=\sum_{j=s-n+1}^{t}\left|\hat{H}_{i j}\right|, i=1, \ldots, n$.

Let us consider a discrete LTI dynamics described by

$x_{k+1}=A x_{k}+E \delta_{k}$, where $x_{k}$ is the state vector, $A$ and $E$ are constant matrices and $A$ is a Schur matrix, and $\delta_{k} \in \Delta=\left\{\delta:\left|\delta-\delta^{\circ}\right| \leq \bar{\delta}\right\}$ with $\delta^{\circ}$ and $\bar{\delta}$ being constant vectors is a disturbance vector.

Definition 2. A set $X \subset \mathbb{R}^{n}$ is called a robust positively invariant (RPI) set for the dynamics (1) iff $A X \oplus E \Delta \subseteq X$.

Definition 3. The minimal RPI (mRPI) set of the dynamics (1) is defined as an RPI set contained in any closed RPI set of the dynamics (1) and the mRPI set is unique and compact.

Theorem 1. ([33,34]) For the system (1) and considering that $A=V \Lambda V^{-1}$ is the Jordan decomposition of $A$, the set

$\Phi(\theta)=\left\{x \in \mathbb{R}^{n}:\left|V^{-1} x\right| \leq(I-|\Lambda|)^{-1}\left|V^{-1} E\right| \bar{\delta}+\theta\right\} \oplus \xi^{\circ}$,

is RPI and attractive for the state trajectories of (1), where $\theta$ being any (arbitrarily small) vector with positive components and $\xi^{\circ}=(I-A)^{-1} E \delta^{\circ}$. It is obtained that

1 for any $\theta$, the set $\Phi(\theta)$ is (positively) invariant, that is, if $x_{0} \in \Phi(\theta)$, then $x_{k} \in \Phi(\theta)$ for all $k \geq 0$;

2 given $\theta \in \mathbb{R}^{n}, \theta>0$, and $x_{0} \in \mathbb{R}^{n}$, there exists $k^{*} \geq 0$ such that $x_{k} \in$ $\Phi(\theta)$ for all $k \geq k^{*}$.

Theorem 2. ([33]) For the system (1) and considering that starting from its initial set denoted as $X_{0}$, the set sequence

$X_{j+1}=A X_{j} \oplus E \Delta, j=0,1,2, \ldots$,

converges to the mRPI set of (1), where $X_{0}$ is an RPI set of (1). Moreover, each iteration of the set sequence is an RPI approximation of the $\mathrm{mRPI}$ set of the system (1).

\section{Problem formulation}

This section introduces the considered discrete LTI system and the design of FDI-oriented SUIO.

\subsection{System model}

Consider the following discrete LTI system affected by multiplicative actuator faults

$x_{k+1}=A x_{k}+B G u_{k}+E \omega_{k}$,

$y_{k}=C x_{k}+F \eta_{k}$,

where $A \in \mathbb{R}^{n \times n}, B=\left[b_{1}, \ldots, b_{i}, \ldots, b_{p}\right] \in \mathbb{R}^{n \times p}$ ( $b_{i}$ is the $i$ th column of $B), E=\left[e_{1}, \ldots e_{i}, \ldots, e_{r}\right] \in \mathbb{R}^{n \times r}\left(e_{i}\right.$ is the $i$ th column of $E), C \in \mathbb{R}^{q \times n}$ and $F \in \mathbb{R}^{q \times s}$ are constant, $k$ denotes the $k$ th time instant, $x_{k} \in \mathbb{R}^{n}$ and $y_{k} \in \mathbb{R}^{q}$ are the state and output vectors, $u_{k}=\left[u_{k}^{1}, \ldots, u_{k}^{i}, \ldots, u_{k}^{p}\right]^{T} \in \mathbb{R}^{p}\left(u_{k}^{i}\right.$ is the $i$ th component of $u_{k}$ generated by the $i$ th actuator) and $\omega_{k} \in \mathbb{R}^{r}$ are known and unknown input vectors, ${ }^{1} \eta_{k} \in \mathbb{R}^{s}$ is the noise vector, and $G$ is a $p \times p$ diagonal matrix modeling actuator faults by $G=\operatorname{diag}\left(f_{1}, \ldots, f_{i}, \ldots, f_{p}\right)$, where $f_{i}$ models the $i$ th actuator. When the $i$ th actuator is healthy, $f_{i}$ is equal to 1 , while if the $i$ th actuator is faulty, $f_{i}$ takes a value inside $[0,1)$, where 0 means that the actuator has completely failed and a value inside $(0,1)$ means that the actuator has partially failed.

Assumption 1. For the system (2), the pair $(A, C)$ is detectable.

Note that we can design a bank of observers to implement the following proposed robust FDI method under Assumption 1.

\footnotetext{
${ }^{1}$ For simplicity, we generally use the term unknown inputs to refer to all unknown signals and factors (including process disturbances, modeling errors, unknown inputs, etc.) affecting the system dynamics in this paper.
} 


\subsection{FDI-oriented design of SUIO}

Following [17], an FDI-oriented SUIO design method is presented here. From (2), an augmented vector is defined as

$v_{k}=\left[\begin{array}{llllllll}f_{1} u_{k}^{1} & f_{2} u_{k}^{2} & \cdots & f_{p} u_{k}^{p} & \omega_{k}^{1} & \omega_{k}^{2} & \cdots & \omega_{k}^{r}\end{array}\right]^{T}$,

where $\omega_{k}^{i}$ is the $i$ th component of $\omega_{k}$ and $v_{k}$ is a $(p+r)$-dimensional vector. Correspondingly, a concatenated parametric matrix matching $v_{k}$ is defined as $B_{E}=\left[\begin{array}{ll}B & E\end{array}\right]$.

Usually, the UIO is designed to be sensitive to faults in some actuators and insensitive to the others. If this feature of UIO is fully employed, it is possible to obtain satisfactory robust FDI results. Thus, in this paper, we propose to divide the augmented vector $v_{k}$ into three subvectors:

$v_{k}=\left[\begin{array}{lll}v_{1, k}^{T} & v_{2, k}^{T} & v_{3, k}^{T}\end{array}\right]^{T}$,

where $v_{1, k} \in \mathbb{R}^{l_{1}}, v_{2, k} \in \mathbb{R}^{l_{2}}$ and $v_{3, k} \in \mathbb{R}^{l_{3}}$ with $l_{1}+l_{2}+l_{3}=p+r$. Note that $v_{1, k}$ includes control inputs that the SUIO does not actively decouple, $v_{2, k}$ includes the other control inputs and some unknown inputs that the SUIO actively decouples while $v_{3, k}$ includes all the remaining unknown inputs that the SUIO does not actively decouple. Moreover, the partitioning of $v_{k}$ in (4) should be done by considering the requirement of robust FDI. ${ }^{2}$ Thus, for robust FDI, we have

$1 \leq l_{1} \leq p-1,1 \leq l_{2} \leq p+r-1,0 \leq l_{3} \leq r$.

Remark 1. From (4), the control input vector $v_{1, k}$ includes fault magnitudes $f_{i}$ for $1 \leq i \leq l_{1}$. Thus, when all the actuators corresponding to $v_{1, k}$ are healthy, we use another notation $\bar{v}_{1, k}$ to represent $v_{1, k}$ such that $v_{1, k}=\mathbf{f} \bar{v}_{1, k}$, where $\mathbf{f}=\operatorname{diag}\left(f_{1}, f_{2}, \ldots f_{l_{1}}\right)$ and $\bar{v}_{1, k}=$ $\left[\begin{array}{llll}u_{k}^{1} & u_{k}^{2} & \cdots & u_{k}^{l_{1}}\end{array}\right]^{T}$.

Assumption 2. The control inputs $u_{k}^{i}$ for $\forall 1 \leq i \leq p$ are bounded. As a result, the control input vector $\bar{v}_{1, k}$ is bounded by a zonotopic set $\bar{U}_{1}$ (i.e., $\bar{v}_{1, k} \in \bar{U}_{1}$ ).

Considering the partitioning of $v_{k}$, the matrix $B_{E}$ can also be rewritten into

$B_{E}=\left[\begin{array}{lll}B_{E}^{1} & B_{E}^{2} & B_{E}^{3}\end{array}\right]$,

where $B_{E}^{1}, B_{E}^{2}$ and $B_{E}^{3}$ correspond to $v_{1, k}, v_{2, k}$ and $v_{3, k}$, respectively. As a result, (2a) can be transformed into

$x_{k+1}=A x_{k}+B_{E}^{1} v_{1, k}+B_{E}^{2} v_{2, k}+B_{E}^{3} v_{3, k}$.

Following the idea mentioned above, under Assumption 1, we design a UIO based on (6) to decouple $v_{2, k}$ as

$z_{k+1}=N z_{k}+T \bar{v}_{1, k}+K y_{k}$,

$\hat{x}_{k}=M z_{k}+H y_{k}$,

$\hat{y}_{k}=C \hat{x}_{k}$,

where $z_{k} \in \mathbb{R}^{n}$ and $\hat{x}_{k} \in \mathbb{R}^{n}$ are the state vector of the UIO and the state estimation vector of the system (2), respectively, $N \in \mathbb{R}^{n \times n}$, $T \in \mathbb{R}^{n \times l_{1}}, K \in \mathbb{R}^{n \times q}, M \in \mathbb{R}^{n \times n}$ and $H \in \mathbb{R}^{n \times q}$ are the parametric matrices of the UIO.

From (6) and (7), the state estimation error vector of the SUIO (7) is defined as

$e_{k}=x_{k}-\hat{x}_{k}$

\footnotetext{
2 We cannot design all the SUIOs to be insensitive or sensitive to faults in all the actuators. But, it is allowed that one or several SUIOs among a bank of SUIOs are insensitive or sensitive to faults in all the actuators. Otherwise, it is impossible to make FI decisions. Thus, for simplicity, we only consider the case $1 \leq l_{1} \leq p-1$.
}

and the dynamics of $e_{k}$ in the healthy situation of all the actuators included in $v_{1, k}$ can be derived as

$$
\begin{aligned}
e_{k+1}= & \Lambda_{0} e_{k}+\Lambda_{1} z_{k}+\Lambda_{2} y_{k}+\Lambda_{3} \bar{v}_{1, k}+\Lambda_{4} v_{2, k} \\
& +\Lambda_{5} v_{3, k}+\Lambda_{6} \eta_{k+1}+\Lambda_{7} \eta_{k}
\end{aligned}
$$

with

$\Lambda_{0}=A-H C A-M K_{1} C$,

$\Lambda_{1}=\left(A-H C A-M K_{1} C\right) M-M N$,

$\Lambda_{2}=\left(A-H C A-M K_{1} C\right) H-M K_{2}$,

$\Lambda_{3}=B_{E}^{1}-M T-H C B_{E}^{1}$,

$\Lambda_{4}=B_{E}^{2}-H C B_{E}^{2}$,

$\Lambda_{5}=B_{E}^{3}-H C B_{E}^{3}$,

$\Lambda_{6}=-H F$,

$\Lambda_{7}=-M K_{1} F$,

$K=K_{1}+K_{2}$.

We can see that $v_{2, k}$ can be decoupled by the UIO if the condition $\Lambda_{4}=\mathbf{0}$ is satisfied. The condition to solve $\Lambda_{4}=\mathbf{0}$ is given in Assumption 3 (see Theorem 1 in [11] for the details).

Assumption 3. The decoupling condition $\operatorname{rank}\left(B_{E}^{2}\right)=\operatorname{rank}\left(C B_{E}^{2}\right)$ of $v_{2, k}$ is satisfied for the equivalent system form (6).

Moreover, by further designing the parametric matrices of the UIO (7) such that $\Lambda_{1}, \Lambda_{2}, \Lambda_{3}$ and $\Lambda_{4}$ are zeros and guaranteeing stability, the dynamics (9) can be reduced into

$e_{k+1}=\Lambda_{0} e_{k}+\Lambda_{5} v_{3, k}+\Lambda_{6} \eta_{k+1}+\Lambda_{7} \eta_{k}$.

In (11), only the unknown inputs included in $v_{2, k}$ are decoupled, while the remaining unknown inputs included in $v_{3, k}$ still affect the system. Thus, in order to implement robust FDI, we have to further consider the effects of $v_{3, k}, \eta_{k+1}$ and $\eta_{k}$ on the residuals. For achieving FDI robustness, we further propose to use the set theory to bound their effects.

Assumption 4. $v_{3, k}$ and $\eta_{k}$ are bounded by $\mathcal{W}_{3}=\left\{v_{3}:\left|v_{3}\right| \leq \bar{v}_{3}\right\}$ and $\mathcal{V}=\{\eta:|\eta| \leq \bar{\eta}\}$, respectively, where both $\bar{v}_{3}$ and $\bar{\eta}$ are known and constant vectors.

Note that the bounding sets $\mathcal{W}_{3}$ and $\mathcal{V}$ can be equivalently transformed into zonotopes with generator matrices denoted by $H_{\bar{v}_{3}}$ and $H_{\bar{\eta}}$, respectively. By considering the zonotopic sets of $v_{3, k}, \eta_{k+1}$ and $\eta_{k}$, a set-based dynamics of (11) is obtained as

$\mathcal{E}_{k+1}=\Lambda_{0} \mathcal{E}_{k} \oplus \Lambda_{5} \mathcal{W}_{3} \oplus \Lambda_{6} \mathcal{V} \oplus \Lambda_{7} \mathcal{V}$,

where $\mathcal{E}_{k}$ is the set of $e_{k}$. It is assumed that the initial condition satisfies $e_{0} \in \mathcal{E}_{0}$. Moreover, in order to implement robust FDI, the residual for the UIO is defined as

$r_{k}=y_{k}-\hat{y}_{k}=C e_{k}+F \eta_{k}$

As a result, the set of $r_{k}$ can be constructed as

$\mathcal{R}_{k}=C \mathcal{E}_{k} \oplus F \mathcal{V}$.

Thus, the UIO and the set theory have been integrated in (12) and (13) (i.e., following the so-called SUIO) for FDI robustness. Since the SUIO is designed to be only sensitive to faults in the actuators included in $v_{1, k}$, faults in the other actuators do not affect the SUIO. Thus, if all actuators corresponding to $v_{1, k}$ are healthy, the following inclusion is guaranteed:

$\mathbf{0} \in \overline{\mathcal{R}}_{k}=\mathcal{R}_{k} \oplus\left\{-r_{k}\right\}$. 
The violation of (14) implies that some of the actuators included in $v_{1, k}$ are faulty. Note that all sets and set operations are based on zonotopes [32]. By using zonotopes, the set-based dynamics composed of (12) and (13) can be equivalently transformed into a center-generator matrix form as

$e_{k+1}^{c}=\Lambda_{0} e_{k}^{c}$,

$H_{k+1}^{e}=\left[\begin{array}{llll}\Lambda_{0} H_{k}^{e} & \Lambda_{5} H_{\bar{v}_{3}} & \Lambda_{6} H_{\bar{\eta}} & \Lambda_{7} H_{\bar{\eta}}\end{array}\right]$,

$r_{k}^{c}=C e_{k}^{c}$,

$H_{k}^{r}=\left[\begin{array}{ll}C H_{k}^{e} & F H_{\bar{\eta}}\end{array}\right]$,

where $e_{k}^{c}, H_{k}^{e}, r_{k}^{c}$ and $H_{k}^{r}$ are the centers and generator matrices of $\mathcal{E}_{k}$ and $\mathcal{R}_{k}$, respectively.

Remark 2. When (15b) is used for iterative computation of zonotopes, in order to avoid the explosion of order of zonotopes, the order-reduction method in Property 3 is used to control the number of columns of $H_{k}^{e}$ in (15) at each step.

\section{Optimal design of FDI-oriented SUIO}

This section designs an optimal FDI-oriented SUIO aiming to reduce the FDI conservatism of single SUIO.

\subsection{Optimal FDI-oriented SUIO}

The shape and size of a zonotope are completely determined by its generator matrix. Because the $F$-norm of a matrix sufficiently considers the effects of all its matrix elements, we use the $F$-norm of the generator matrix of a zonotope to describe its size here (see $[32,35])$. The optimality of the SUIO is based on the zonotope size minimization [35]. Thus, the $F$-norm based size of $\mathcal{E}_{k+1}$ is given as $\left\|\mathcal{E}_{k+1}\right\|_{F}=\left\|H_{k+1}^{e}\right\|_{F}$ leading to

$$
\begin{aligned}
\left\|\mathcal{E}_{k+1}\right\|_{F}^{2} & =\left\|H_{k+1}^{e}\right\|_{F}^{2}=\operatorname{tr}\left(H_{k+1}^{e}\left(H_{k+1}^{e}\right)^{T}\right) \\
& =\operatorname{tr}\left(\Lambda_{0}^{T} \Lambda_{0} H_{k}^{e}\left(H_{k}^{e}\right)^{T}\right)+\operatorname{tr}(U)
\end{aligned}
$$

with

$$
\begin{aligned}
\bar{M}= & M K_{1}, \\
U= & H C B_{E}^{3} H_{\bar{v}_{3}}\left(H_{\bar{v}_{3}}\right)^{T}\left(B_{E}^{3}\right)^{T} C^{T} H^{T}+\bar{M} F H_{\bar{\eta}} H_{\bar{\eta}}^{T} F^{T} \bar{M}^{T} \\
& -H C B_{E}^{3} H_{\bar{v}_{3}}\left(H_{\bar{v}_{3}}\right)^{T}\left(B_{E}^{3}\right)^{T}-B_{E}^{3} H_{\bar{v}_{3}}\left(H_{\bar{v}_{3}}\right)^{T}\left(B_{E}^{3}\right)^{T} C^{T} H^{T} \\
& +B_{E}^{3} H_{\bar{v}_{3}}\left(H_{\bar{v}_{3}}\right)^{T}\left(B_{E}^{3}\right)^{T}+H F H_{\bar{\eta}} H_{\bar{\eta}}^{T} F^{T} H^{T} .
\end{aligned}
$$

According to (10e), the priority of designing the matrix $H$ is to satisfy $\Lambda_{4}=0$ such that $v_{2, k}$ is actively decoupled. This implies that in (16), only $\bar{M}$ can be tuned to improve the FDI performance. Moreover, the optimal value $\bar{M}^{*}$ of $\bar{M}$ can be obtained by solving the following equation:

$$
\frac{\partial\left(\left\|\mathcal{E}_{k+1}\right\|_{F}^{2}\right)}{\partial \bar{M}}=\mathbf{0}
$$

where since the optimal value $\bar{M}^{*}$ is time-varying, we use a symbol $\bar{M}_{k}^{*}$ to denote its value at time instant $k$ as

$$
\begin{aligned}
\bar{M}_{k}^{*}= & (I-H C) A H_{k}^{e}\left(H_{k}^{e}\right)^{T} C^{T} \\
& \times\left(F H_{\bar{\eta}} H_{\bar{\eta}}^{T} F^{T}+C H_{k}^{e}\left(H_{k}^{e}\right)^{T} C^{T}\right)^{-1} .
\end{aligned}
$$

By observing (17a), we know that $M$ is a free parameter. Thus, we set $M$ as the identity matrix such that $\bar{M}=K_{1}$ for brevity. Conse- quently, corresponding to $\bar{M}_{k}^{*}$, a group of the optimal time-varying parametric matrices for (7) can be obtained:

$$
\begin{aligned}
& H^{*}= B_{E}^{2}\left[\left(C B_{E}^{2}\right)^{T} C B_{E}^{2}\right]^{-1}\left(C B_{E}^{2}\right)^{T} \\
&+H_{0}\left\{I-C B_{E}^{2}\left[\left(C B_{E}^{2}\right)^{T} C B_{E}^{2}\right]^{-1}\left(C B_{E}^{2}\right)^{T}\right\}, \\
& K_{1, k}^{*}=(I-H C) A H_{k}^{e}\left(H_{k}^{e}\right)^{T} C^{T} \\
& \times\left(F H_{\bar{\eta}} H_{\bar{\eta}}^{T} F^{T}+C H_{k}^{e}\left(H_{k}^{e}\right)^{T} C^{T}\right)^{-1}, \\
& K_{2, k}^{*}=\left(A-H^{*} C A-\bar{M}_{k}^{*}\right) H^{*} \\
& N_{k}^{*}=A- H^{*} C A-\bar{M}_{k}^{*} C \\
& T_{k}^{*}=B_{E}^{1}-H^{*} C B_{E}^{1},
\end{aligned}
$$

where the optimal parametric matrices are obtained by designing $\Lambda_{1}, \Lambda_{2}, \Lambda_{3}$ and $\Lambda_{4}$ in (10) to be zeros and $H_{0}$ is an arbitrarily given matrix with proper dimensions.

\subsection{Set separation-based guaranteed FD condition}

From (12) and (13), the size of $\mathcal{R}_{k}$ mainly depends on the generator matrix $H_{k}^{e}$. Moreover, the group of optimal parametric matrices in (20) also depend on $H_{k}^{e}$. Thus, once the initial zonotope $\mathcal{E}_{0}$ with the center $e_{0}^{c}$ and generator matrix $H_{0}^{e}$ is given, the evolution of the set-based dynamics (12) and (13) is fixed.

However, because the optimal parametric matrices are actually time-varying, it leads to that (11) becomes a linear time-varying (LTV) system instead of an LTI system. Thus, the results of Theorem 1 does not apply and it is difficult to construct an RPI set for (11) to describe the steady-state behaviors of (12) and further establish an RPI set separation-based FD condition. This paper proposes to construct an over-approximate dynamics with time-invariant parametric matrices for (12) to approximately describe its steadystate behaviors. This approximate dynamics is used to establish an RPI set separation-based FD condition for guaranteed robust FDI using the optimal SUIOs.

Theorem 3. For the system (2), the SUIO (7) with a group of optimal matrices given in (19) and (20), and the initial condition $e_{0} \in \mathcal{E}_{0}$, there always exists a proper $\lambda(\lambda \geq 1)$ that allows to find a set $\overline{\mathcal{E}}=\lambda \tilde{\mathcal{E}}$ such that $e_{k} \in \overline{\mathcal{E}}$ for $\forall k \geq k^{*}$, where the set $\tilde{\mathcal{E}}$ is an RPI set of the dynamics $e_{k+1}=\Lambda_{0, k^{*}} e_{k}+\Lambda_{5, k^{*}} v_{3, k}+\Lambda_{6, k^{*}} \eta_{k+1}+\Lambda_{7, k^{*}} \eta_{k}$ with

$\Lambda_{0, k^{*}}=A-H^{*} C A-\bar{M}_{k^{*}}^{*} C$,

$\Lambda_{5, k^{*}}=B_{E}^{3}-H^{*} C B_{E}^{3}$,

$\Lambda_{6, k^{*}}=-H^{*} F$,

$\Lambda_{7, k^{*}}=-\bar{M}_{k^{*}}^{*} F$

and $k^{*}$ is an arbitrarily given value with $k^{*} \geq 0$.

Proof. As given in (19), once $e_{0} \in \mathcal{E}_{0}$ is given (i.e., the generator matrix $H_{0}^{e}$ of $\mathcal{E}_{0}$ is given), the trajectory of $\bar{M}_{k}^{*}$ is fixed for all $k \geq 0$. If we give an integer $k^{*}$, the time line could be divided into two segments: $0 \leq k \leq k^{*}$ and $k>k^{*}$. Consequently, the evolution of the set-based dynamics (12) can also be divided into two segments: $\mathcal{E}_{k}$ $\left(0 \leq k \leq k^{*}\right)$ and $\mathcal{E}_{k}\left(k>k^{*}\right)$.

Furthermore, by considering the value of $\bar{M}_{k}^{*}$ at time instant $k=k^{*}$ (i.e., $\bar{M}_{k^{*}}^{*}$ ), we can obtain the following dynamics with constant parametric matrices

$e_{k+1}=\Lambda_{0, k^{*}} e_{k}+\Lambda_{5, k^{*}} v_{3, k}+\Lambda_{6, k^{*}} \eta_{k+1}+\Lambda_{7, k^{*}} \eta_{k}$

and the corresponding set-based dynamics

$\tilde{\mathcal{E}}_{k+1}=\Lambda_{0, k^{*}} \tilde{\mathcal{E}}_{k} \oplus \Lambda_{5, k^{*}} \mathcal{W}_{3} \oplus \Lambda_{6, k^{*}} \mathcal{V} \oplus \Lambda_{7, k^{*}} \mathcal{V}$, 
where $\tilde{\mathcal{E}}_{k}$ denotes the set of $e_{k}$ in (22) at time instant $k$.

Similarly, considering the two segments of time $0 \leq k \leq k^{*}$ and $k>k^{*}$, we can construct a new set trajectory as

$\hat{\mathcal{E}}_{k}= \begin{cases}\mathcal{E}_{k} & 0 \leq k \leq k^{*}, \\ \tilde{\mathcal{E}}_{k} & k>k^{*} .\end{cases}$

Since $\bar{M}_{k^{*}}^{*}$ is only optimal for the dynamics (12) at time instant $k=k^{*}$, it is not optimal for the set-based dynamics (23) for all $k>k^{*}$. Thus, by comparing the two set trajectories $\mathcal{E}_{k}(k \geq 0)$ and $\hat{\mathcal{E}}_{k}(k \geq 0)$, we have

$\left\|\mathcal{E}_{k}\right\|_{F}^{2}<\left\|\hat{\mathcal{E}}_{k}\right\|_{F}^{2}$ for $k>k^{*}$.

Under Assumption 4, according to (12) and (23), both $\mathcal{E}_{\infty}$ and $\hat{\mathcal{E}}_{\infty}$ are centered at the origin if $\mathcal{E}_{0}$ is centered at the origin. This means that $\mathcal{E}_{\infty}$ and $\hat{\mathcal{E}}_{\infty}$ intersect with each other. Furthermore, even though the condition $\left\|\mathcal{E}_{\infty}\right\|_{F}^{2}<\left\|\hat{\mathcal{E}}_{\infty}\right\|_{F}^{2}$ cannot guarantee the inclusion $\mathcal{E}_{\infty} \subseteq \hat{\mathcal{E}}_{\infty}$, at least, this can guarantee that $\mathcal{E}_{\infty}$ and $\hat{\mathcal{E}}_{\infty}$ almost overlap with each other.

According to the notion of RPI sets, the set $\hat{\mathcal{E}}_{\infty}$ is actually the minimal RPI (mRPI) set of the dynamics (22). Generally, although the mRPI set cannot be obtained, we can construct an RPI set $\tilde{\mathcal{E}}$ to contain $\hat{\mathcal{E}}_{\infty}$ (i.e., $\hat{\mathcal{E}}_{\infty} \subseteq \tilde{\mathcal{E}}$ ). This means that the RPI set $\tilde{\mathcal{E}}$ has a larger possibility to contain $\mathcal{E}_{\infty}$. Since the scaling of an RPI set is still an RPI set for the same dynamics, we can construct a new and larger RPI set $\overline{\mathcal{E}}=\lambda \tilde{\mathcal{E}}(\lambda \geq 1)$ for (22). Simultaneously, as long as the scaler $\lambda$ is sufficiently large, it is always possible to find a value of $\lambda$ such that $\mathcal{E}_{\infty} \subseteq \overline{\mathcal{E}}=\lambda \tilde{\mathcal{E}}$.

This also means that as $k$ tends to $\infty, \mathcal{E}_{k}$ will enter into $\overline{\mathcal{E}}$ at a time instant. It is known that, as long as the initial condition $e_{0} \in \mathcal{E}_{0}, \mathcal{E}_{k}$ is the set of $e_{k}$ (i.e., $e_{k} \in \mathcal{E}_{k}$ for $\forall k \geq 0$ ). Thus, we know that after a certain time instant, $e_{k}$ will enter into the set $\overline{\mathcal{E}}$. Thus, the proof is complete.

Remark 3. In Theorem $3, k^{*}$ should be set as a large value to construct the set $\overline{\mathcal{E}}$. Moreover, as $k^{*}$ tends to $\infty$ and $\lambda$ approaches to 1 , it increases the chance to obtain a set $\overline{\mathcal{E}}$ to sufficiently approximate $\mathcal{E}_{\infty}$. Thus, in the following theorem, we further propose a method to obtain a proper $k^{*}$ able to provide sufficiently high approximate precision for the establishment of set separation-based guaranteed FD conditions.

Theorem 4. Given the system (2), the zonotopic iterative equation (15b) and the SUIO (7) with a group of optimal matrices given in (19) and (20), let $P_{k^{*}}=H_{k^{*}}^{e}\left(H_{k^{*}}^{e}\right)^{T}$ for $\forall k^{*} \geq 0$. Denoting $P=P_{\infty}$ and $\bar{M}^{*}=$ $\bar{M}_{\infty}^{*}$, one has

$$
\begin{aligned}
P= & \left(A-H^{*} C A\right)\left(P-P C^{T}\left(C P C^{T}+F Q_{\eta} F^{T}\right)^{-1} C P\right) \\
& \times\left(A-H^{*} C A\right)^{T}+Q,
\end{aligned}
$$

$\bar{M}^{*}=\left(A-H^{*} C A\right) P C^{T}\left(C P C^{T}+F Q_{\eta} F^{T}\right)^{-1}$,

where $Q=\Lambda_{5} Q_{\bar{v}_{3}} \Lambda_{5}^{T}+\Lambda_{6} Q_{\bar{\eta}} \Lambda_{6}^{T}, Q_{\eta}=H_{\bar{\eta}} H_{\bar{\eta}}^{T}$, and $Q_{\bar{v}_{3}}=H_{\bar{v}_{3}}\left(H_{\bar{v}_{3}}\right)^{T}$.

Proof. With (15b) and $P_{k^{*}+1}=H_{k^{*}+1}^{e}\left(H_{k^{*}+1}^{e}\right)^{T}$, we have

$P_{k^{*}+1}=\Lambda_{0} P_{k^{*}} \Lambda_{0}^{T}+\Lambda_{5} Q_{\bar{v}_{3}} \Lambda_{5}^{T}+\Lambda_{6} Q_{\bar{\eta}} \Lambda_{6}^{T}+\Lambda_{7} Q_{\bar{\eta}} \Lambda_{7}^{T}$.

With the notation of $Q$ and by using $\Lambda_{0}$ and $\Lambda_{7}$ given in (21a) and $(21 \mathrm{~d})$, we obtain

$$
\begin{aligned}
& P_{k^{*}+1}=\left(A-H^{*} C A-\bar{M}_{k^{*}}^{*} C\right) P_{k^{*}}\left(A-H^{*} C A-\bar{M}_{k^{*}}^{*} C\right)^{T} \\
& +\bar{M}_{k^{*}}^{*} F Q_{\bar{\eta}} F^{T}\left(\bar{M}_{k^{*}}^{*}\right)^{T}+Q .
\end{aligned}
$$

Besides, considering that $\bar{M}_{k^{*}}^{*}$ is a solution of (19), we obtain
$\bar{M}_{k^{*}}^{*}\left(C P C^{T}+F Q_{\eta} F^{T}\right)=\left(A-H^{*} C A\right) P C^{T}$,

and multiplying $\left(\bar{M}_{k^{*}}^{*}\right)^{T}$ on the right side of the equation above, we have

$\bar{M}_{k^{*}}^{*}\left(C P C^{T}+F Q_{\eta} F^{T}\right)\left(\bar{M}_{k^{*}}^{*}\right)^{T}=\left(A-H^{*} C A\right) P C^{T}\left(\bar{M}_{k^{*}}^{*}\right)^{T}$.

By substituting (28) into (27), it can be obtained that

$P_{k^{*}+1}=\left(A-H^{*} C A\right)\left(P_{k^{*}}-P_{k^{*}} C^{T}\left(C P_{k^{*}} C^{T}+F Q_{\eta} F^{T}\right)^{-1} C P_{k^{*}}\right)$

$$
\left(A-H^{*} C A\right)^{T}+Q \text {. }
$$

Based on (20) and at $k^{*}=\infty$, we have $P_{k^{*}+1}=P_{k^{*}}=P$ and $\bar{M}_{k^{*}+1}^{*}=$ $\bar{M}_{k^{*}}^{*}=\bar{M}^{*}$. Hence, by using (28) and (29), (26) can be obtained. Thus, the proof is completed. $\square$

Remark 4. From Theorem $4, \bar{M}_{k^{*}}^{*}$ converges to a constant value as $k^{*}$ tends to $\infty$, which satisfies (26). However, (26) is a Ricatti equation. Thus, we consider solving it in an iterative way and finding an approximate value of $\bar{M}^{*}$ with sufficiently high precision by iterating (29). Correspondingly, a proper $k^{*}$ can be obtained for Theorem 3 to compute a less conservative set $\overline{\mathcal{E}}$ of $e_{k}$ at steady state.

According to the results above, we provide an algorithm to obtain an approximate value of $\bar{M}^{*}$ with a precision $\epsilon$ and the corresponding $k^{*}$ in Algorithm 4.

\section{Algorithm 4. Approximation of $\bar{M}^{*}$ with precision $\epsilon$}

1: $\quad$ Provide an initial set $\mathcal{E}_{0}$ (i.e., $H_{0}^{e}$ is given);

2: $\quad$ Obtain $P_{0}=H_{0}^{e}\left(H_{0}^{e}\right)^{T}$;

3: $\quad$ Establish an approximate precision $\epsilon$;

4: $\quad$ Iterate (29) with the initial value $P_{0}$;

5: $\quad$ Define a criterion $P_{k^{*}+1}-P_{k^{*}} \leq \epsilon \mathbf{I}$;

6: $\quad$ Iterate (29) to obtain a proper $k^{*}$;

7: $\quad$ Obtain an approximation of $\bar{M}^{*}$ with precision $\epsilon$;

8: $\quad$ Construct $\overline{\mathcal{E}}$ in Theorem 3;

9: $\quad$ return $k^{*}, \bar{M}_{k^{*}}^{*}$ and $\overline{\mathcal{E}}$;

Note that the dynamics of state estimation error vector $e_{k}$ in (11) correspond to the healthy situation of the actuators included in $v_{1, k}$. If there are faults in the actuators included in $v_{1, k}$ (i.e., there are fault magnitudes $f_{i}$ in $v_{1, k}$ that are not 1 ), the dynamics (9) is changed into

$$
\begin{aligned}
e_{k+1}^{f}= & \Lambda_{0} e_{k}^{f}+\Lambda_{1} z_{k}+\Lambda_{2} y_{k}+\Lambda_{4} v_{2, k} \\
& +\Lambda_{5} v_{3, k}+\left(B_{E}^{1} \mathbf{f}-M T-H C B_{E}^{1} \mathbf{f}\right) \bar{v}_{1, k} \\
& +\Lambda_{6} \eta_{k+1}+\Lambda_{7} \eta_{k},
\end{aligned}
$$

where $e_{k}^{f}$ denotes the state estimation error vector of the SUIO (7) in the faulty situation. Under the conditions that $\Lambda_{1}, \Lambda_{2}, \Lambda_{3}$ and $\Lambda_{4}$ in (10) are zeros, the dynamics (30) is reduced into

$$
\begin{aligned}
e_{k+1}^{f}= & \Lambda_{0} e_{k}^{f}+\left(B_{E}^{1} \mathbf{f}-M T-H C B_{E}^{1} \mathbf{f}\right) \bar{v}_{1, k} \\
& +\Lambda_{5} v_{3, k}+\Lambda_{6} \eta_{k+1}+\Lambda_{7} \eta_{k} .
\end{aligned}
$$

Similar to Theorems 3 and 4, a proper time instant $k_{f}^{*}$ is selected (i.e., $\bar{M}_{k_{f}^{*}}^{*}$ ) to construct an approximate dynamics

$$
\begin{aligned}
e_{k+1}^{f}= & \Lambda_{0, k_{f}^{*}} e_{k}^{f}+\left(B_{E}^{1} \mathbf{f}-M T-H C B_{E}^{1} \mathbf{f}\right) \bar{v}_{1, k} \\
& +\Lambda_{5, k_{f}^{*}} v_{3, k}+\Lambda_{6, k_{f}^{*}} \eta_{k+1}+\Lambda_{7, k_{f}^{*}} \eta_{k} .
\end{aligned}
$$

Under Assumption 2, an RPI set can be constructed for $e_{k}^{f}$ by using (32). By means of Theorems 3 and 4, this RPI set can be used to construct a set $\overline{\mathcal{E}}_{f}$ to contain $e_{k}^{f}$ at steady state of the corresponding faulty situation. Thus, a set separation-based FD condition for the SUIO (7) is provided by Theorem 5 . 
Theorem 5. For the system (2) and the UIO (7) with a group of optimal matrices given in (20), if a fault $\mathbf{f}$ in the actuators corresponding to the input vector $v_{1, k}$ occurs, then this fault $\mathbf{f}$ can be detected under the condition $\overline{\mathcal{R}} \cap \overline{\mathcal{R}}_{f}=\emptyset$, where $\overline{\mathcal{R}}=C \overline{\mathcal{E}} \oplus F \mathcal{V}$ and $\overline{\mathcal{R}}_{f}=C \overline{\mathcal{E}}_{f} \oplus F \mathcal{V}$.

Proof. The proof is straightforward and omitted here. $\square$

\section{Optimal guaranteed robust FDI}

This section proposes an optimal guaranteed robust FDI approach, which involves two layers. The first layer is devoted to designing single optimal SUIO as presented in Section 4. The second layer focuses on an optimal observer configuration for a bank of optimal SUIOs, which is presented in this section.

\subsection{Partitioning of Control Inputs}

In Section 4, an optimal SUIO is designed to be sensitive to faults in $l_{1}$ actuators and insensitive to faults in the other $p-l_{1}$ actuators. Here, we consider the use of a bank of SUIOs to implement robust FDI as follows: if we design each SUIO to be only sensitive to faults in a different group of actuators, it is possible for the bank of optimal SUIOs to implement both robust FD and FI. This is the idea of the proposed FDI approach.

Since there are $p$ actuators equipped in the system, theoretically, we have $\mathcal{N}_{l_{1}}=C_{p}^{l_{1}}$ ways to design an SUIO to be only sensitive to faults in $l_{1}$ actuators. Considering that $l_{1}$ can take $p-1$ different values (i.e., $l_{1}=1,2, \ldots, p-1$ ), the total number of possible optimal FDI-oriented SUIOs for the system (2) is obtained as

$\mathbf{N}_{T}=\mathcal{N}_{1}+\mathcal{N}_{2}+\cdots+\mathcal{N}_{p-2}+\mathcal{N}_{p-1}$,

where, as given above, $\mathcal{N}_{l_{1}}=C_{p}^{l_{1}}$ for all $1 \leq l_{1} \leq p-1$.

To explain the proposed approach, we label the $p$ actuators with the indices from 1 to $p$, respectively, and define an index set to describe a combination of $l_{1}$ actuators. In total, there are $\mathcal{N}_{l_{1}}$ index sets and these $\mathcal{N}_{l_{1}}$ index sets together form another set $S_{l_{1}}$ describing all the $\mathcal{N}_{l_{1}}$ combinations of $l_{1}$ actuators as

$S_{l_{1}}=\left\{J_{1}^{l_{1}}, J_{2}^{l_{1}}, \ldots, J_{j}^{l_{1}}, \ldots, J_{\mathcal{N}_{l_{1}}}^{l_{1}}\right\}$,

where $J_{j}^{l_{1}}$ is the $j$ th index set describing a combination of $l_{1}$ actuators with $1 \leq j \leq \mathcal{N}_{l_{1}}$ and is denoted as

$J_{j}^{l_{1}}=\left\{J_{j, 1}^{l_{1}}, J_{j, 2}^{l_{1}}, \ldots, J_{j, t}^{l_{1}}, \ldots, J_{j, l_{1}}^{l_{1}}\right\}$,

where $t$ denotes the index of the actuator $J_{j, t}^{l_{1}}$ among the combination $J_{j}^{l_{1}}$ with $1 \leq J_{j, t}^{l_{1}} \leq p$ and $1 \leq t \leq l_{1}$.

Example 1. We consider a system with 4 actuators to explain the different notations defined above. In this example, we could have $1 \leq l_{1} \leq 3$ with $\mathcal{N}_{1}=C_{4}^{1}=4, \mathcal{N}_{2}=C_{4}^{2}=6, \mathcal{N}_{3}=C_{4}^{3}=4$ and $\mathbf{N}_{T}=14$. When designing SUIOs to be sensitive to faults in 2 actuators, the index set $S_{2}=\left\{J_{1}^{2}, J_{2}^{2}, J_{3}^{2}, J_{4}^{2}, J_{5}^{2}, J_{6}^{2}\right\}$ with $J_{1}^{2}=\{1,2\}\left(J_{1,1}^{2}=1\right.$ and $\left.J_{1,2}^{2}=2\right), J_{2}^{2}=\{1,3\}\left(J_{2,1}^{2}=1\right.$ and $\left.J_{2,2}^{2}=3\right), J_{3}^{2}=\{1,4\}\left(J_{3,1}^{2}=1\right.$ and $\left.J_{3,2}^{2}=4\right), J_{4}^{2}=\{2,3\}\left(J_{4,1}^{2}=2\right.$ and $\left.J_{4,2}^{2}=3\right), J_{5}^{2}=\{2,4\}\left(J_{5,1}^{2}=2\right.$ and $\left.J_{5,2}^{2}=4\right)$ and $J_{6}^{2}=\{3,4\}\left(J_{6,1}^{2}=3\right.$ and $\left.J_{6,2}^{2}=4\right)$. By using the same idea above, the index sets $S_{1}$ and $S_{3}$ can be obtained as well.

\subsection{Partitioning of unknown inputs}

In Section 5.1, partitioning of control inputs is considered in detail to obtain an optimal observer configuration for detection and isolation of faults in all the system actuators. However, in the case of robust FDI, we still need to further analyze the effect of unknown inputs to reduce the FDI conservatism as much as possible from the viewpoint of FDI performance.
In Section 4, when designing an optimal SUIO for the system (2), all the unknown inputs are divided into two groups, where the first group is included in $v_{2, k}$ and the other group is included in $v_{3, k}$. According to the idea in Section 4, the first group is arranged to be actively decoupled by the SUIO, while the second group includes the remaining unknown inputs that the SUIO is unable to further actively decouple. Actually, in the proposed design, all the unknown inputs are expected to be decoupled (i.e., let $v_{3, k}$ be empty). In this way, we are able to achieve not only robust FDI but also the least FDI conservatism with respect to the unknown inputs. However, due to the limitation of active decoupling ability of the SUIO, in general, we have to leave some unknown inputs into $v_{3, k}$ and then passively consider their effect by using the set-theoretic methods.

Assumption 5. Each single unknown input included in $\omega_{k}$ has the same effect on the sizes of residual sets.

Assumption 6. Without loss of generality, it is assumed that all the outputs of the system (2) are independent of each other.

Theoretically, we could obtain the situation in Assumption 5 by adjusting the parametric matrix $E$ of $\omega_{k}$ in (2). Thus, based on the partitioning of control inputs included in $v_{1, k}$ (see Section 5.1), our main objective here is to optimize the number of unknown inputs included in $v_{3, k}$ as small as possible. According to [11], the number of unknown inputs that a UIO can actively decouple is not larger than the number of independent system outputs. If we consider designing an optimal SUIO to be sensitive to faults in $l_{1}$ actuators, it means that the number of control inputs included in $v_{2, k}$ that the SUIO needs to actively decouple is $p-l_{1}$. Thus, under Assumption 6, we can know that if $p-l_{1}>q$, the SUIO cannot decouple any unknown input (i.e., $v_{2, k}$ cannot include any unknown input and all the unknown inputs should be included in $v_{3, k}$ ), i.e., $1 \leq l_{1} \leq p-q-1$.

This implies that when analyzing the effect of unknown inputs on robust FDI, the two cases below are considered:

- Case $1\left(1 \leq l_{1} \leq p-q-1\right)$ : there do not exist SUIOs that is able to decouple all the remaining $p-l_{1}$ control inputs included in $v_{2, k}$. As a result, we cannot design optimal SUIOs for this case. Thus, this case is omitted;

- Case $2\left(p-q \leq l_{1} \leq p-1\right)$ : In this case, it is possible to decouple all the remaining $p-l_{1}$ control inputs included in $v_{2, k}$ and some unknown inputs. As a result, we should analyze the effect of unknown inputs on the FDI conservatism for each value of $l_{1}$ and obtain the shortest (i.e., optimal) vector $v_{3, k}$ of each value of $l_{1}$ for optimal robust FDI.

Thus, from the optimal FDI performance point of view, we mainly need to analyze Case 2 . If considering different values of $l_{1}$ in Case 2 , there are totally $q$ situations (i.e., each value of $l_{1}$ corresponds to one situation) that should be analyzed. Moreover, for each situation, we have $\mathcal{N}_{l_{1}}$ combinations of $l_{1}$ control inputs. Thus, the total number of combinations of $l_{1}$ control inputs in Case 2 is obtained as

$\mathbf{N}_{U}=\mathcal{N}_{p-q}+\mathcal{N}_{p-q+1}+\cdots+\mathcal{N}_{p-2}+\mathcal{N}_{p-1}$.

In order to explain the idea optimizing $v_{3, k}$ for each combination, we consider the combination $J_{j}^{l_{1}}$ included in $S_{l_{1}}$ (see (34)) as an example. In this combination, the control inputs in $u_{k}$ are partitioned into two subvectors: one corresponding to $v_{1, k}$ including $l_{1}$ control inputs and the other one corresponding to $v_{2, k}$ including $p-l_{1}$ control inputs. For convenience, these first and second subvectors are denoted as $u_{k}^{J_{1}^{l_{1}}}$ and $u_{k}^{\bar{l}_{j}}$, respectively and the partitioning of the input matrix $B$ corresponding to $u_{k}^{J_{j}^{l_{1}}}$ and $u_{k}^{\bar{J}_{j}}$ is 
denoted as $B^{l_{1}^{l_{1}}}$ and $B^{\bar{l}_{j}}{ }^{l_{1}}$, respectively. This means that the vector $B u_{k}$ is divided into $B^{J^{l_{1}}} u_{k}^{J_{j}^{l_{1}}}$ and $B^{I_{j}^{l_{1}}} u_{k}^{\bar{J}_{j}^{l_{j}}}$.

Similar to the analysis of control inputs in Section 5.1 and the contents above, we also need to partition the unknown input vector $\omega_{k}$. First, the number of unknown inputs included in $v_{2, k}$ is denoted as $l_{u}$ such that $l_{u}=r-l_{3}$. If we want to decouple $v_{2, k}, l_{2}=p-l_{1}+l_{u} \leq q$ should be satisfied. As a result, we have

$1 \leq l_{u} \leq q-p+l_{1}$.

Thus, we should select $l_{u}$ unknown inputs out of $r$ unknown inputs of $\omega_{k}$. Theoretically, the number of combinations of $l_{u}$ unknown inputs is $\mathcal{U}_{l_{u}}=C_{r}^{l_{u}}$. As shown in (37), $l_{u}$ can take $q-p+l_{1}$ values, each value corresponding to one situation. Thus, there are $q-p+l_{1}$ situations considering all values $1 \leq l_{u} \leq q-p+l_{1}$ and each situation includes $\mathcal{U}_{l_{u}}=C_{r}^{l_{u}}$ combinations. Consequently, for the system (2), the total number of combinations to select $l_{u}$ unknown inputs for $v_{2, k}$ is denoted as

$\mathbf{U}_{T}=\mathcal{U}_{1}+\mathcal{U}_{2}+\cdots+\mathcal{U}_{l_{u}}+\cdots+\mathcal{U}_{q-p+l_{1}}$.

Similar to Section 5.1, the $r$ unknown inputs included in $\omega_{k}$ are labelled by the indices from 1 to $r$, respectively. Similar to the set $S_{l_{1}}$, we define an index set to describe a combination of $l_{u}$ unknown inputs. Totally, there are $\mathcal{U}_{l_{u}}$ index sets for one situation selecting $l_{u}$ unknown inputs and we define another set $T_{l_{u}}$ to describe all the $\mathcal{U}_{l_{u}}$ combinations as

$T_{l_{u}}=\left\{I_{1}^{l_{u}}, I_{2}^{l_{u}}, \ldots, I_{i}^{l_{u}}, \ldots, I_{\mathcal{U}_{l_{u}}}^{l_{u}}\right\}$,

where $I_{i}^{l_{u}}$ is the $i$ th index set describing a combination of $l_{u}$ unknown inputs with $1 \leq i \leq \mathcal{U}_{l_{u}}$ and

$I_{i}^{l_{u}}=\left\{I_{i, 1}^{l_{u}}, I_{i, 2}^{l_{u}}, \ldots, I_{i, \bar{t}}^{l_{u}}, \ldots, I_{i, l_{u}}^{l_{u}}\right\}$,

where $\bar{t}$ denotes the index of the unknown input $I_{i, \bar{t}}^{l_{u}}$ among the combination $I_{i}^{l_{u}}$ with $1 \leq I_{i, \bar{t}}^{l_{u}} \leq r$ and $1 \leq \bar{t} \leq l_{u}$.

Without loss of generality, we consider the combination $I_{i}^{l_{u}}$ in (40) as an example to show the partitioning of $\omega_{k}$ for simplicity. In this case, we select $l_{u}$ unknown inputs to compose the vector $v_{2, k}$ and then design an optimal FDI-oriented SUIO to decouple $v_{2, k}$ for FDI. In this combination, all the unknown inputs in $\omega_{k}$ are divided into two groups: the first one is denoted as $\omega_{k}^{i}$ i while the second one is denoted as $\omega_{k}^{\bar{I}_{i}^{l}}$. Correspondingly, the vector $E \omega_{k}$ is divided into $E^{I_{i}^{l_{u}}} \omega_{k}^{I_{i}^{l_{u}}}$ and $E^{\bar{I}_{i}^{l_{u}}} \omega_{k}^{\bar{I}_{i}^{l_{u}}}$.

Based on the aforementioned analysis on the combination $J_{j}^{l_{1}}$ of control inputs and the combination $I_{i}^{l_{u}}$ of unknown inputs, a partitioning of all the control inputs and unknown inputs as shown in (4) and (5) can be obtained (i.e., forming a group of $v_{1, k}, v_{2, k}, v_{3, k}$, $B_{E}^{1}, B_{E}^{2}$ and $B_{E}^{3}$ as shown in (6)):

$v_{1, k}=u_{k}^{J_{1}^{l}}$,

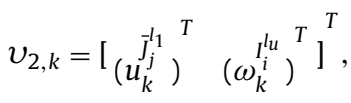

$v_{3, k}=\omega_{k}^{\bar{I}_{i}^{l} u}$,

$B_{E}^{1}=B^{J_{j}^{l_{1}}}$

$B_{E}^{2}=\left[\begin{array}{ll}{ }_{B}^{J_{j}^{1}} & E^{l_{i}^{l_{u}}}\end{array}\right]$,

$B_{E}^{3}=E^{I_{i}^{l}}$.

Furthermore, we need to verify whether the partitioning of control and unknown inputs in (41) allows the design of the SUIOs. This verification can be done by testing the decoupling condition given in Assumption 3. If $B_{E}^{2}$ obtained in (41e) satisfies its corresponding decoupling condition in Assumption 3, it is considered that this partitioning can be used for the FDI-oriented SUIOs (i.e., $v_{2, k}$ obtained in (41b) may be actively decoupled). Otherwise, the partitioning in (41) is discarded. We see that a partitioning of control and unknown inputs is determined by a pair of $J_{j}^{l_{1}}$ and $I_{i}^{l_{u}}$. Thus, we use a pair $\left(J_{j}^{l_{1}}\right.$, $\left.I_{i}^{l_{u}}\right)$ to denote a partitioning of $u_{k}$ and $\omega_{k}$ for brevity.

As analyzed and explained above, for each element of $S_{l_{1}}$ for all $p-q \leq l_{1} \leq p-1$, we can obtain all its corresponding partitionings $\left(J_{j}^{l_{1}}, I_{i}^{l_{u}}\right)$ enabling the design of the SUIOs and simultaneously including the largest number of unknown inputs into $v_{2, k}$ (i.e., $l_{u}$ takes its allowed largest value). However, the detection and isolation of faults in all the four actuators are based on a precondition that any SUIO out of a bank of SUIOs can guarantee the detection of faults occurring in the actuators whose control inputs are sensed by this SUIO. Thus, all the obtained optimal partitionings $\left(J_{j}^{l_{1}}, I_{i}^{l_{u}}\right)$ should further satisfy the set separation-based guaranteed robust FDI condition in Theorem 5. Moreover, we use the notations $\overline{\mathcal{R}}^{J^{l_{1}}, I_{i}^{l^{l}}}$ and $\overline{\mathcal{R}}_{f}^{J_{1}^{l_{1}}, I_{i}^{l^{l}} \text { to }}$ to denote the healthy and faulty sets of the partitioning $\left(J_{j}^{l_{1}}, I_{i}^{l_{u}}\right)$.

As a result, with respect to $u_{k}$ and $\omega_{k}$, all the obtained optimal partitionings $\left(J_{j}^{l_{1}}, I_{i}^{l_{u}}\right)$ owning the FDI-oriented SUIOs and satisfying their corresponding guaranteed robust FDI conditions are collected into a set $\mathcal{O}$. Thus, from this optimal FDI viewpoint, robust FDI should be implemented by choosing a group of optimal partitionings of control and unknown inputs out of the set $\mathcal{O}$, designing a bank of optimal FDI-oriented SUIOs for them (each SUIO for an optimal partitioning) and analyzing the residuals of this bank of SUIOs. The whole design method and procedure above are summarized in Algorithm 5. Note that as the number of control and unknown inputs/system dimensions increases, the complexity of Algorithm 5 also increases. However, due to that all the analysis done in Algorithm 5 is offline, this increase of complexity originated from the number of control and unknown inputs/system dimensions does not significantly increase the online computational complexity of the proposed robust FDI method. For example, we could use Matlab codes to implement the offline analysis in Algorithm 5 and effectively handle this kind of complexity increase induced by the number of control and unknown inputs/system dimensions.

Algorithm 5. Partitioning of control and unknown inputs

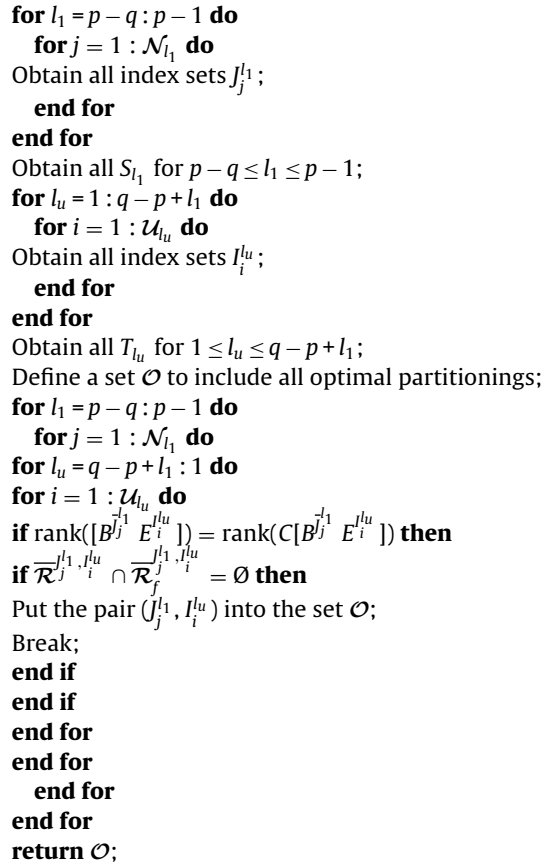


Example 2. This example considers $p=4$ actuators, $r=3$ unknown inputs and $q=3$ independent outputs to explain the method partitioning the control and unknown inputs. According to the proposed method, Case 2 classified in Section 3.1 has $1 \leq l_{1} \leq 3$ and $\mathbf{N}_{T}=14$ :

- when $l_{1}=1: S_{1}=\left\{J_{1}^{1}, J_{2}^{1}, J_{3}^{1}, J_{4}^{1}\right\}$ with $J_{1}^{1}=\{1\}\left(J_{1,1}^{1}=1\right)$ and $\bar{J}_{1}^{1}=$ $\{2,3,4\}, J_{2}^{1}=\{2\}\left(J_{2,1}^{1}=2\right)$ and $\bar{J}_{2}^{1}=\{1,3,4\}, J_{3}^{1}=\{3\}\left(J_{3,1}^{1}=3\right)$ and $\bar{J}_{3}^{1}=\{1,2,4\}$, and $J_{4}^{1}=\{4\}\left(J_{4,1}^{1}=4\right)$ and $\bar{J}_{4}^{1}=\{1,2,3\}$;

- when $l_{1}=2: S_{2}=\left\{J_{1}^{2}, J_{2}^{2}, J_{3}^{2}, J_{4}^{2}, J_{5}^{2}, J_{6}^{2}\right\}$ with $J_{1}^{2}=\{1,2\}\left(J_{1,1}^{2}=1\right.$ and $\left.J_{1,2}^{2}=2\right)$ and $\bar{J}_{1}^{2}=\{3,4\}, J_{2}^{2}=\{1,3\}\left(J_{2,1}^{2}=1\right.$ and $\left.J_{2,2}^{2}=3\right)$ and $\bar{J}_{2}^{2}=$ $\{2,4\}, J_{3}^{2}=\{1,4\}\left(J_{3,1}^{2}=1\right.$ and $\left.J_{3,2}^{2}=4\right)$ and $\bar{J}_{3}^{2}=\{2,3\}, J_{4}^{2}=\{2,3\}$ $\left(J_{4,1}^{2}=2\right.$ and $\left.J_{4,2}^{2}=3\right)$ and $\bar{J}_{4}^{2}=\{1,4\}, J_{5}^{2}=\{2,4\}\left(J_{5,1}^{2}=2\right.$ and $J_{5,2}^{2}=$ 4) and $\bar{J}_{5}^{2}=\{1,3\}$, and $J_{6}^{2}=\{3,4\}\left(J_{6,1}^{2}=3\right.$ and $\left.J_{6,2}^{2}=4\right)$ and $\bar{J}_{6}^{2}=$ $\{1,2\}$;

- when $l_{1}=3: S_{3}=\left\{J_{1}^{3}, J_{2}^{3}, J_{3}^{3}, J_{4}^{3}\right\}$ with $J_{1}^{3}=\{1,2,3\}\left(J_{1,1}^{3}=1, J_{1,2}^{3}=2\right.$ and $\left.J_{1,3}^{3}=3\right)$ and $\bar{J}_{1}^{3}=\{4\}, J_{2}^{3}=\{1,2,4\}\left(J_{2,1}^{3}=1, J_{2,2}^{3}=2\right.$ and $J_{2,3}^{3}=$ 4) and $\bar{J}_{2}^{3}=\{3\}, J_{3}^{3}=\{1,3,4\}\left(J_{3,1}^{3}=1, J_{3,2}^{3}=3\right.$ and $\left.J_{3,3}^{3}=4\right)$ and $\bar{J}_{3}^{3}=\{2\}, J_{4}^{3}=\{2,3,4\}\left(J_{4,1}^{3}=2, J_{4,2}^{3}=3\right.$ and $\left.J_{4,3}^{3}=4\right)$ and $\bar{J}_{4}^{3}=\{1\}$.

Moreover, taking the combination $J_{2}^{2}=\{1,3\}$ as an example, we have $u_{k}^{J_{2}^{2}}=v_{1, k}=\left[\begin{array}{lll}f_{1} u_{k}^{1} & f_{3} u_{k}^{3}\end{array}\right]^{T}, u_{k}^{\bar{J}_{2}^{2}}=\left[\begin{array}{lll}f_{2} u_{k}^{2} & f_{4} u_{k}^{4}\end{array}\right]^{T}, B^{J_{2}^{2}}=$ $\left[\begin{array}{ll}b_{1} & b_{3}\end{array}\right]$ and $B^{J_{2}^{2}}=\left[\begin{array}{ll}b_{2} & b_{4}\end{array}\right]$ for explaining the meaning of $u_{k}^{J_{1}^{1}}, u_{k}^{\bar{J}_{j}^{1}}$, $B^{J^{l_{1}}}$ and $B^{\bar{J}_{j}^{l_{1}}}$.

Furthermore, according to the idea partitioning the unknown inputs above, for the combination $l_{1}=1, l_{u}$ takes no values, which is ignored. For the combination $l_{1}=2$, we have $l_{u}=1$. For the combination $l_{1}=3$, we have $1 \leq l_{u} \leq 2$.

Without loss of generality, we take $l_{1}=3$ and $1 \leq l_{u} \leq 2$ as an example to show the idea partitioning the unknown inputs above. Thus, we have $\mathcal{U}_{1}=3, \mathcal{U}_{2}=3$ and $\mathcal{U}_{T}=6$ with

- when $l_{u}=1: T_{1}=\left\{I_{1}^{1}, I_{2}^{1}, I_{3}^{1}\right\}$ with $I_{1}^{1}=\{1\}\left(I_{1,1}^{1}=1\right)$ and $\bar{I}_{1}^{1}=\{2,3\}$, $I_{2}^{1}=\{2\}\left(I_{2,1}^{1}=2\right)$ and $\bar{I}_{2}^{1}=\{1,3\}$, and $I_{3}^{1}=\{3\}\left(I_{3,1}^{1}=3\right)$ and $\bar{I}_{3}^{1}=$ $\{1,2\}$;

- when $l_{u}=2: I_{2}=\left\{I_{1}^{2}, I_{2}^{2}, I_{3}^{2}\right\}$ with $I_{1}^{2}=\{1,2\}\left(I_{1,1}^{2}=1\right.$ and $\left.I_{1,2}^{2}=2\right)$ and $\bar{I}_{1}^{2}=\{3\}, I_{2}^{2}=\{1,3\}\left(I_{2,1}^{2}=1\right.$ and $\left.I_{2,2}^{2}=3\right)$ and $\bar{I}_{2}^{2}=\{2\}$, and $I_{3}^{2}=$ $\{2,3\}\left(I_{3,1}^{2}=2\right.$ and $\left.I_{3,2}^{2}=3\right)$ and $\bar{I}_{3}^{2}=\{1\}$.

Similarly, considering the combination $I_{2}^{1}=\{2\}$ as an example, we have $\omega_{k}^{I_{2}^{1}}=\omega_{k}^{2}, \omega_{k}^{I_{2}^{1}}=\left[\begin{array}{ll}\omega_{k}^{1} & \omega_{k}^{3}\end{array}\right]^{T}, E^{I_{2}^{1}}=\left[e_{2}\right]$ and $E^{\bar{I}_{2}^{1}}=\left[\begin{array}{ll}e_{1} & e_{3}\end{array}\right]$ for explaining the meaning of $\omega_{k}^{I_{i}^{l}}, \omega_{k}^{I_{i}^{I_{u}}}, E^{I_{i}^{I^{u}}}$ and $E^{\bar{I}_{i}^{I^{l}}}$. Thus, when $l_{1}=3$ and $1 \leq l_{u} \leq 2$, we could obtain the optimal partitionings of control and unknown inputs:

- Case $1\left(J_{1}^{3}=\{1,2,3\}\right.$ and $\left.1 \leq l_{u} \leq 2\right)$ : based on the analysis above, we should test the criterion $\operatorname{rank}\left(\left[B^{I_{1}^{3}} E_{i}^{I_{i}^{l_{u}}}\right]\right)=\operatorname{rank}\left(C\left[B^{I_{1}^{3}} E_{i}^{I_{i}^{l_{u}}}\right]\right)$ for all $1 \leq l_{u} \leq 2$ and $1 \leq i \leq \mathcal{U}_{l_{u}}$ to obtain the shortest vector $\omega_{k}^{I^{l_{u}}}$ matching $u_{k}^{\bar{J}_{j}^{1}}$ to compose an optimal vector $v_{2, k}$;

- Case $2\left(J_{2}^{3}=\{1,2,4\}\right.$ and $\left.1 \leq l_{u} \leq 2\right)$, Case $3\left(J_{3}^{3}=\{1,3,4\}\right.$ and $\left.1 \leq l_{u} \leq 2\right)$ and Case $4\left(J_{4}^{3}=\{2,3,4\}\right.$ and $\left.1 \leq l_{u} \leq 2\right)$ : similar idea to Case 1 can be used for these three cases to obtain their optimal partitionings of control and unknown inputs, respectively.

Remark 5. For each combination above, the optimal partitionings of control and unknown inputs may not be unique (i.e., the shortest vector $v_{3, k}$ may not be unique). Thus, for all combinations included in $S_{l_{1}}$ for $p-q \leq l_{1} \leq p-1$, we define a set $\mathcal{O}$ to contain all their optimal partitionings (described by pairs $\left(J_{j}^{l_{1}}, I_{i}^{l_{u}}\right)$ ) together. Furthermore, from the optimal FDI performance point of view, we could select a group of pairs out of the set $\mathcal{O}$ to design a bank of optimal SUIOs, where each SUIO is designed to actively decouple the vector $v_{2, k}$ described by an optimal pair. As a result of the optimal partitionings of control and unknown inputs, we can make full use of the potential FDI performance of the FDI scheme using a bank of SUIOs.

\subsection{Optimal guaranteed robust FDI algorithm}

The set $\mathcal{O}$ includes all the optimal partitionings of control and unknown inputs for the system (2). This implies that we need to design the optimal configuration of observers by selecting a group of pairs $\left(J_{j}^{l_{1}}, I_{i}^{l_{u}}\right)$ out of the set $\mathcal{O}$. Then, we further design a bank of optimal SUIOs to be sensitive to the control inputs included in $v_{1, k}$ and insensitive to $v_{2, k}$, where each SUIO matches a pair of optimal partitioning of control and unknown inputs of the designed optimal observer configuration.

For convenience, we make a classification of all the elements of the set $\mathcal{O}$. For each pair $\left(J_{j}^{l_{1}}, I_{i}^{l_{u}}\right)$ in the set $\mathcal{O}$, it corresponds to a group of $v_{1, k}, v_{2, k}$ and $v_{3, k}$. Thus, we collect all the pairs in $\mathcal{O}$ that has the same number of unknown inputs included in $v_{3, k}$ into a new set $\mathcal{O}_{l_{3}}$, as defined in Section 3.2, $l_{3}$ denotes the number of unknown inputs included in $v_{3, k}$ of these pairs. Based on this classification, all the pairs of the set $\mathcal{O}$ can be divided into several small sets $\mathcal{O}_{l_{3}}$ with $1 \leq l_{3} \leq l_{3, \max }\left(l_{3, \max }\right.$ denotes the maximal value of $l_{3}$ among all the pairs in the set $\mathcal{O}$ ). For the set $\mathcal{O}, l_{3}$ may not be able to take all the integers of the interval $\left[1, l_{3, \max }\right]$. However, for brevity of explaining the proposed optimal robust FDI algorithm, we assume that $l_{3}$ can take all integers inside $\left[1, l_{3, \max }\right]$ and thus the set $\mathcal{O}$ can be equivalently denoted as a set of a group of the sets $\mathcal{O}_{l_{3}}$, i.e.,

$\mathcal{O}=\left\{\mathcal{O}_{l_{3}}: 1 \leq l_{3} \leq l_{3, \max }\right\}$.

In order to reduce the FDI conservatism as much as possible, we should select the pairs out of $\mathcal{O}_{l_{3}}$ with smaller $l_{3}$ in priority (i.e., the criterion to assess the optimality of an observer configuration). Thus, we first analyze the pairs in the set $\mathcal{O}_{1}$. If we can select a group of pairs out of $\mathcal{O}_{1}$ to design a bank of SUIOs able to detect and isolate faults in all $p$ actuators, we can take these SUIOs as the final observer configuration. Otherwise, we need to continue to select some other pairs out of $\mathcal{O}_{2}$ together with the pairs from $\mathcal{O}_{1}$ to design a bank of SUIOs for detection and isolation of faults in all the actuators. Thus, the worst case is to finally consider the pairs from $\mathcal{O}_{1_{3, \max }}$. Consequently, in this way, we could design a bank of optimal SUIOs sensitive to the least unknown inputs and could simultaneously detect and isolate faults in all the actuators. Eventually, we could use this optimal observer configuration to design a bank of optimal SUIOs to achieve the FDI optimality.

Example 3. In order to help the readers understand the proposed idea above, we consider again Example 2 in Section 3.2 to explain the proposed optimal guaranteed FDI algorithm. In this case, it is assumed that the set $\mathcal{O}$ is obtained as

$\mathcal{O}=\left\{\mathcal{O}_{1}, \mathcal{O}_{2}\right\}$

with $\mathcal{O}_{1}=\{(\{1,2,3\},\{1,2\}),(\{1,2,4\},\{1,3\})\}$ and $\mathcal{O}_{2}=\{(\{1,2\}$, $\{3\}),(\{3,4\},\{1\}),(\{2,3\},\{2\})\}$.

According to the proposed idea above, we first consider the elements of the set $\mathcal{O}_{1}$. Thus, if designing two SUIOs 1 and 2 to match the pairs $(\{1,2,3\},\{1,2\})$ and $(\{1,2,4\},\{1,3\})$, respectively, we can obtain a fault signature matrix in Table 1(a) (note that the element 1 in Table 1 means that the SUIO can detect faults in the actuator, while the element 0 means the opposite). By observing the fault 


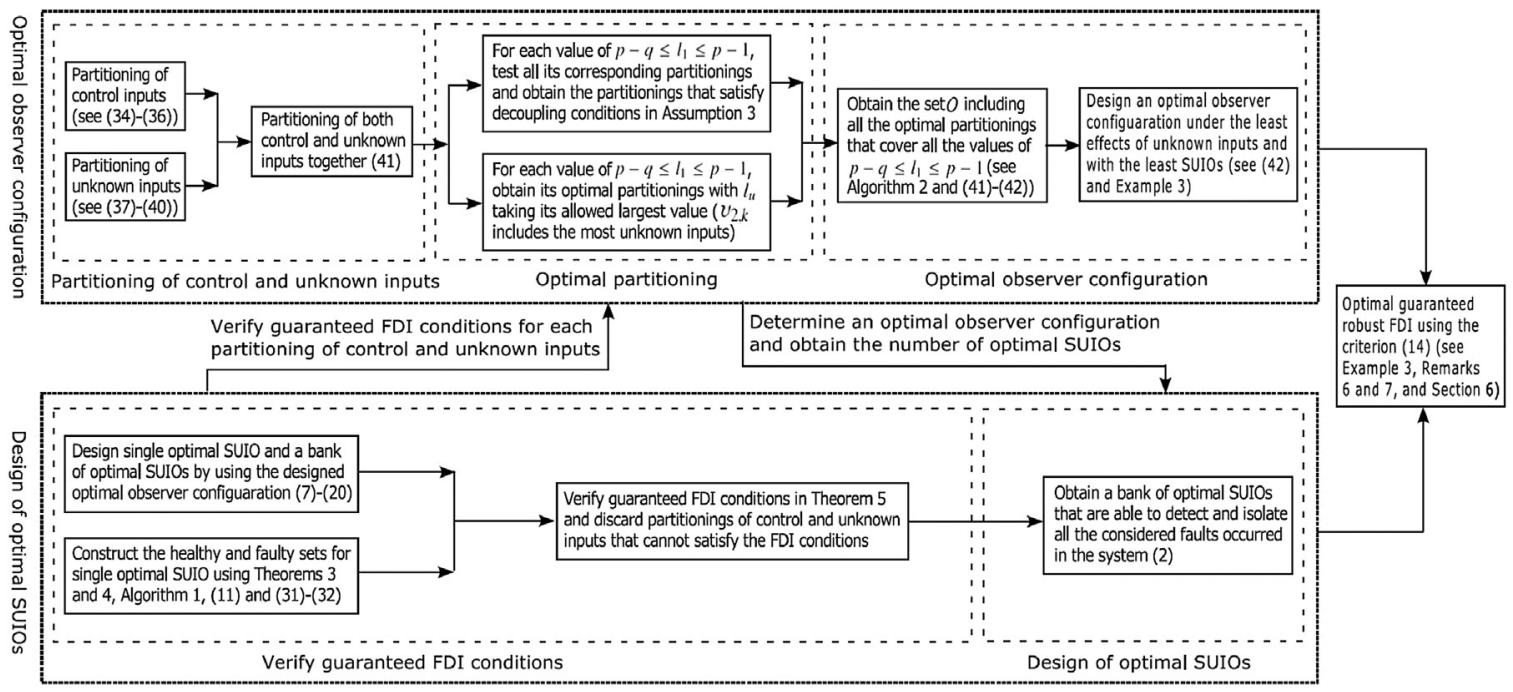

Fig. 1. Logical flow chart of optimal robust FDI method.

Table 1

SUIO partitionings of Example 3.

\begin{tabular}{|c|c|c|c|c|c|}
\hline \multirow[t]{3}{*}{ Faults } & \multicolumn{5}{|c|}{ SUIOs } \\
\hline & \multicolumn{2}{|c|}{ (a) } & \multicolumn{3}{|c|}{ (b) } \\
\hline & 1 & 2 & 1 & 2 & 3 \\
\hline In Actuator 1 & 1 & 1 & 1 & 1 & 1 \\
\hline In Actuator 2 & 1 & 1 & 1 & 1 & 1 \\
\hline In Actuator 3 & 1 & 0 & 1 & 0 & 0 \\
\hline In Actuator 4 & 0 & 1 & 0 & 1 & 0 \\
\hline
\end{tabular}

Faults SUIOs

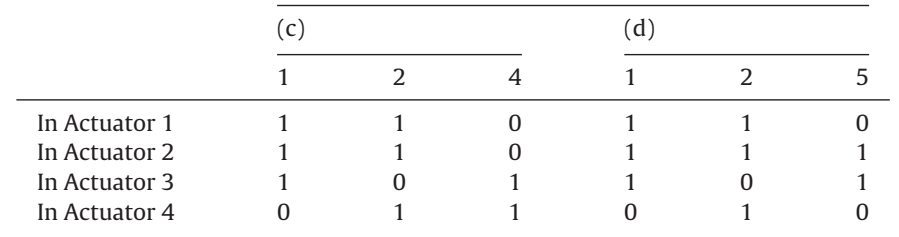

signature matrix in Table 1(a), it is known that faults in the actuators 1 and 2 can be distinguished from faults in the actuators 3 and 4 while faults in the actuators 1 and 2 cannot be distinguished from each other.

This means that for isolating faults in the actuators 1 and 2, we need more SUIOs and should further analyze the pairs in $\mathcal{O}_{2}$. Thus, if designing SUIOs 3,4 and 5 matching the pairs $(\{1,2\},\{3\}),(\{3$, $4\},\{1\})$ and $(\{2,3\},\{2\})$, respectively, and select an SUIO out of the SUIOs 3, 4 and 5 to work with the SUIOs 1 and 2 together for robust FDI, we can further obtain fault signature matrices in Table 1(b)-(d). By analyzing the fault signature matrices in Table 1, we can see that the observer configuration in Table 1 (d) can be used to distinguish faults in all the four actuators. As a result, it can be concluded that the SUIOs 1, 2 and 5 could be the optimal observer configuration in this example. Moreover, its optimality consists in that this observer configuration needs the least SUIOs for robust FDI of all the actuators and the SUIOs are affected by the least unknown inputs. Besides, all the three SUIOs are also designed to be optimal SUIOs as already introduced in Section 4.1.

In Example 3, if we cannot find an SUIO from the set $\mathcal{O}_{2}$ to form the optimal three-observer FDI configuration, the proposed method further finds another SUIO from the set $\mathcal{O}_{2}$ to form an optimal four-observer FDI configuration. Theoretically, the procedure to find an optimal observer configuration for robust FDI of all the actuators ends until all the SUIOs from the set $\mathcal{O}$ is completely considered.

Remark 6. The criterion to choose an optimal observer configuration includes two aspects. Under Assumption 5, the first aspect consists in minimizing the total number of unknown inputs included in a bank of optimal SUIOs together (i.e., for each SUIO out of a bank of SUIOs, we have the corresponding number of unknown inputs in the corresponding vector $v_{3, k}$. The minimization objective is to obtain the least total number of unknown inputs of all SUIOs included in their corresponding vectors $v_{3, k}$ ). The second aspect consists in minimizing the number of SUIOs included in an observer configuration. This means that if there exist several observer configurations that have the same total number of unknown inputs, we further choose an observer configuration out of them such that the chosen observer configuration has the least number of observers. It should be mentioned that the former is prior to the latter. Additionally, for a particular robust FDI application, the chosen observer configuration based on these two rules may not be unique and we could select any optimal observer configuration among them as the final optimal observer configuration for the implementation of the robust FDI application.

Remark 7. As shown in Section 5.3, an optimal observer configuration provides a fault signature matrix as in Table 1(d), where faults in each actuator are labelled by a unique fault signature vector. Thus, online, the proposed FDI algorithm makes FD testing (14) for all the SUIOs and composes a real-time fault signature vector. As a result, a fault is detected if the criterion (14) is violated for an SUIO. Furthermore, the fault can be isolated by matching the realtime fault signature vector with the off-line obtained fault signature matrix.

Additionally, in order to help the readers accurately understand the contributions of this paper, we also present a logical flow chart in Fig. 1 to summarize the whole implementation of the proposed robust FDI method including the procedures of designing optimal SUIO and optimal observer configuration. 


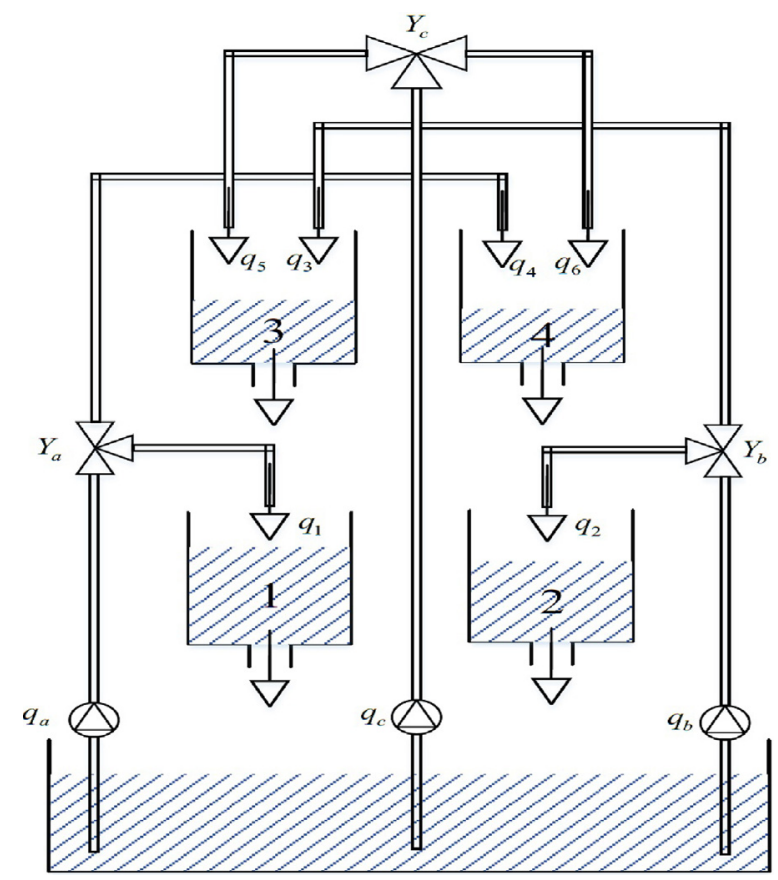

Fig. 2. The four-tank system taken from [36].

\section{Application: the four-tank system}

This section presents the application of the proposed FDI method to a four-tank system as shown in Fig. 2. The four-tank system can be approximated by the nonlinear model [36]:

$\begin{aligned} \frac{d h_{1}}{d t} & =-\frac{a_{1}}{A} \sqrt{2 g h_{1}}+\frac{a_{3}}{A} \sqrt{2 g h_{3}}+\frac{r_{1}}{3600 A} q_{a}, \\ \frac{d h_{2}}{d t} & =-\frac{a_{2}}{A} \sqrt{2 g h_{2}}+\frac{a_{4}}{A} \sqrt{2 g h_{4}}+\frac{r_{2}}{3600 A} q_{b}, \\ \frac{d h_{3}}{d t} & =-\frac{a_{3}}{A} \sqrt{2 g h_{3}}+\frac{1-r_{2}}{3600 A} q_{b}+\frac{r}{3600 A} q_{c}, \\ \frac{d h_{4}}{d t} & =-\frac{a_{4}}{A} \sqrt{2 g h_{4}}+\frac{1-r_{1}}{3600 A} q_{a}+\frac{1-r}{3600 A} q_{c},\end{aligned}$

where $q_{j}$ and $h_{i}$ satisfy the following physical constraints: $Q_{j}^{\min } \leq$ $q_{j} \leq Q_{j}^{\max }(j=a, b, c)$ and $H_{i}^{\min } \leq h_{i} \leq H_{i}^{\max }(i=1,2,3,4)$, where $Q_{C}^{\min }=0, Q_{C}^{\max }=2.5, r=0.5$, and the values of the other parameters of the four-tank system can be found in [36]. The inputs are the water flows through the three pumps denoted as $q_{a}, q_{b}$ and $q_{c}$ and the states are the water levels of the four tanks $\left(h_{1}, h_{2}, h_{3}\right.$ and $\left.h_{4}\right)$.

Considering the convenience of linearizing operation, we define new state variables $x_{i}=\sqrt{h_{i}}(i=1,2,3,4)$ and the original nonlinear model can be transformed into

$$
\begin{aligned}
\frac{d x_{1}}{d t} & =-\frac{a_{1}}{A} \sqrt{\frac{g}{2}}+\frac{a_{3}}{A} \sqrt{\frac{g}{2}} \frac{x_{3}}{x_{1}}+\frac{r_{1} q_{a}}{7200 A} \frac{1}{x_{1}}, \\
\frac{d x_{2}}{d t} & =-\frac{a_{2}}{A} \sqrt{\frac{g}{2}}+\frac{a_{4}}{A} \sqrt{\frac{g}{2}} \frac{x_{4}}{x_{2}}+\frac{r_{2} q_{b}}{7200 A} \frac{1}{x_{2}}, \\
\frac{d x_{3}}{d t} & =-\frac{a_{3}}{A} \sqrt{\frac{g}{2}}+\frac{1-r_{2}}{7200 A} \frac{q_{b}}{x_{3}}+\frac{r q_{c}}{7200 A} \frac{1}{x_{3}}, \\
\frac{d x_{4}}{d t} & =-\frac{a_{4}}{A} \sqrt{\frac{g}{2}}+\frac{1-r_{1}}{7200 A} \frac{q_{a}}{x_{4}}+\frac{1-r}{7200 A} \frac{q_{c}}{x_{4}}
\end{aligned}
$$

In order to verify the proposed FDI method, we can further obtain a linearization model around the equilibrium point defined by $x_{i}^{0}$ and $q_{j}^{0}$ with $i=1,2,3,4$ and $j=a, b, c\left(q_{c}^{0}=0\right.$ and the values of the other parameters are displayed in [36]) by using the Jacobian method. Thus, the new states of the linearized model are given as $\Delta x_{i}=x_{i}-x_{i}^{0}=\sqrt{h_{i}}-\sqrt{h_{i}^{0}}$ and the inputs are $\Delta q_{j}=q_{j}-q_{j}^{0}$, where $i=1,2,3,4$ and $j=a, b, c$. The linearized model is obtained as

$$
\begin{aligned}
\Delta \dot{x}_{1}= & \left(-\frac{a_{3} x_{3}^{0}}{A} \sqrt{\frac{g}{2}}-\frac{r_{1} q_{a}^{0}}{7200 A}\right) \frac{\Delta x_{1}}{\left(x_{1}^{0}\right)^{2}}+\frac{a_{3}}{A} \sqrt{\frac{g}{2}} \frac{\Delta x_{3}}{x_{1}^{0}}+\frac{r_{1}}{7200 A} \frac{\Delta q_{a}}{x_{1}^{0}}, \\
\Delta \dot{x}_{2}= & \left(-\frac{a_{4} x_{4}^{0}}{A} \sqrt{\frac{g}{2}}-\frac{r_{2} q_{b}^{0}}{7200 A}\right) \frac{\Delta x_{2}}{\left(x_{2}^{0}\right)^{2}}+\frac{a_{4}}{A} \sqrt{\frac{g}{2}} \frac{\Delta x_{4}}{x_{2}^{0}}+\frac{r_{2}}{7200 A} \frac{\Delta q_{b}}{x_{2}^{0}}, \\
\Delta \dot{x}_{3}= & \left(r_{2} q_{b}^{0}-q_{b}^{0}-r q_{c}^{0}\right) \frac{\Delta x_{3}}{7200 A\left(x_{3}^{0}\right)^{2}}+\frac{1-r_{2}}{7200 A} \frac{\Delta q_{b}}{x_{3}^{0}}+\frac{r}{7200 A} \frac{\Delta q_{c}}{x_{3}^{0}}, \\
\Delta \dot{x}_{4}= & \left(r_{1} q_{a}^{0}-q_{a}^{0}+r q_{c}^{0}-q_{c}^{0}\right) \frac{\Delta x_{4}}{7200 A\left(x_{4}^{0}\right)^{2}}+\frac{1-r_{1}}{7200 A} \frac{\Delta q_{a}}{x_{4}^{0}}+\frac{1-r}{7200 A} \frac{\Delta q_{c}}{x_{4}^{0}} .
\end{aligned}
$$

Furthermore, in order to obtain a discrete-time linear model from the above continuous-time linear model around the equilibrium point $x_{i}^{0}$ and $q_{j}^{0}$ with $i=1,2,3,4$ and $j=a, b, c$, we take the first-order forward Euler difference method to implement the discretization and the sampling period $T_{S}$ is set as $T_{S}=10 \mathrm{~s}$. For simplicity, in the following simulations, we use the $k$-th step to represent the time instant $t=k T_{s}$. For the convenience of simulation, we consider that the outputs of the discrete-time linear model are the system states. We assume that all the outputs can be measured with some sensors equipped in the four-tank system under the effects of measurement noises.

Therefore, the resulting matrices of the linear model are obtained as

$A=\left[\begin{array}{cccc}0.8769 & 0 & 0.0869 & 0 \\ 0 & 0.8581 & 0 & 0.0838 \\ 0 & 0 & 0.9111 & 0 \\ 0 & 0 & 0 & 0.9159\end{array}\right]$,
$B=\left[\begin{array}{ccc}0.0175 & 0 & 0 \\ 0 & 0.0232 & 0 \\ 0 & 0.0351 & 0.0293 \\ 0.0407 & 0 & 0.0291\end{array}\right]$ and
$C=\left[\begin{array}{llll}1 & 0 & 0 & 0 \\ 0 & 1 & 0 & 0 \\ 0 & 0 & 1 & 0 \\ 0 & 0 & 0 & 1\end{array}\right]$. The distribution matrices of the unknown inputs in $\omega_{k}$ and the measurement noises in $\eta_{k}$ are given as $E=$ $\left[\begin{array}{llll}0.7142 & 0.5310 & 0.4978 & 0.2404\end{array}\right]$

$\begin{array}{llll}0.3080 & 0.7151 & 0.9360 & 0.6849\end{array}$

$\begin{array}{llll}0.6712 & 0.5048 & 0.3893 & 0.8393\end{array}$ and

$\left[\begin{array}{llll}0.6524 & 0.4880 & 0.1171 & 0.9701\end{array}\right]$

$F=\left[\begin{array}{llll}0.2152 & 0.5100 & 0.3020 & 0.7753 \\ 0.7603 & 0.4956 & 0.0896 & 0.1794 \\ 0.5841 & 0.6514 & 0.8260 & 0.1094 \\ 0.4030 & 0.7437 & 0.3896 & 0.9052\end{array}\right]$.

It is given that

$\omega_{k} \in \mathcal{W}=\left[\begin{array}{l}0 \\ 0 \\ 0 \\ 0\end{array}\right] \oplus\left[\begin{array}{cccc}0.001 & 0 & 0 & 0 \\ 0 & 0.001 & 0 & 0 \\ 0 & 0 & 0.001 & 0 \\ 0 & 0 & 0 & 0.001\end{array}\right] \mathbb{B}^{4}$ and

$\eta_{k} \in \mathcal{V}=\left[\begin{array}{l}0 \\ 0 \\ 0 \\ 0\end{array}\right] \oplus\left[\begin{array}{cccc}0.001 & 0 & 0 & 0 \\ 0 & 0.001 & 0 & 0 \\ 0 & 0 & 0.001 & 0 \\ 0 & 0 & 0 & 0.001\end{array}\right] \mathbb{B}^{4}$

The inputs $u_{j}=q_{j}-q_{j}^{0},(j=a, b, c)$ are bounded by

$\left[\begin{array}{l}u_{a} \\ u_{b} \\ u_{c}\end{array}\right] \in U=\left[\begin{array}{c}1.5 \\ 1 \\ 2\end{array}\right] \oplus\left[\begin{array}{ccc}0.1 & 0 & 0 \\ 0 & 0.1 & 0 \\ 0 & 0 & 0.1\end{array}\right] \mathbb{B}^{3}$.

In this example, there are three control inputs, four system outputs and four unknown inputs. Thus, based on the proposed method, the set $\mathcal{O}$ is obtained as $\mathcal{O}=\left\{\mathcal{O}_{1}, \mathcal{O}_{2}, \mathcal{O}_{3}\right\}$ with 


$$
\begin{aligned}
\mathcal{O}_{1}= & \{(\{1,2,3\},\{1,2,3\}),(\{1,2,3\},\{2,3,4\}), \\
& (\{1,2,3\},\{1,2,4\}),(\{1,2,3\},\{1,3,4\})\}, \\
\mathcal{O}_{2}= & \{(\{1,2\},\{1,2\}),(\{1,2\},\{1,3\}),(\{1,2\},\{1,4\}), \\
& (\{1,2\},\{2,3\}),(\{1,2\},\{2,4\}),(\{1,2\},\{3,4\}), \\
& (\{1,3\},\{1,2\}),(\{1,3\},\{1,3\}),(\{1,3\},\{1,4\}), \\
& (\{1,3\},\{2,3\}),(\{1,3\},\{2,4\}),(\{1,3\},\{3,4\}), \\
& (\{2,3\},\{1,2\}),(\{2,3\},\{1,3\}),(\{2,3\},\{1,4\}), \\
& (\{2,3\},\{2,3\}),(\{2,3\},\{2,4\}),(\{2,3\},\{3,4\})\}, \\
\mathcal{O}_{3}= & \{(\{1\},\{1\}),(\{1\},\{2\}),(\{1\},\{3\}),(\{1\},\{4\}), \\
& (\{2\},\{1\}),(\{2\},\{2\}),(\{2\},\{3\}),(\{2\},\{4\}), \\
& (\{3\},\{1\}),(\{3\},\{2\}),(\{3\},\{3\}),(\{3\},\{4\})\} .
\end{aligned}
$$

In order to illustrate the effectiveness of the proposed method, we consider three fault scenarios:

- Scenario I: the first actuator (the left pump in Fig. 2) is faulty, while the other actuators (the middle and right pumps) are healthy, i.e., $f_{1}=0.3, f_{2}=1$ and $f_{3}=1$;

- Scenario II: the second actuator (the right pump in Fig. 2) is faulty, while the other actuators (the middle and left pumps) are healthy, i.e., $f_{1}=1, f_{2}=0.6$ and $f_{3}=1$;

- Scenario III: the third actuator (the middle pump in Fig. 2) is faulty, while the other actuators (the left and right pumps) are healthy, i.e., $f_{1}=1, f_{2}=1$ and $f_{3}=0.7$.

Our objective is to design a bank of optimal SUIOs with the smallest number of unknown inputs included in $v_{3, k}$ to reduce the FDI conservatism, and the designed SUIOs should satisfy guaranteed FDI conditions. Moreover, we should decrease the number of SUIOs as small as possible to reduce the complexity of the proposed FDI algorithm. Therefore, under the guaranteed FDI conditions, the optimal SUIO configuration pairs are

(a) $\left\{\left(\{1,2\},\left\{a^{1}, a^{2}\right\}\right),\left(\{2,3\},\left\{a^{3}, a^{4}\right\}\right)\right\}, a^{i}=1,2,3,4, a^{i} \neq a^{j}(i, j=1$, $2,3,4, i \neq j)$

(b) $\left\{\left(\{1,2\},\left\{b^{1}, b^{2}\right\}\right),\left(\{1,3\},\left\{b^{3}, b^{4}\right\}\right)\right\}, b^{i}=1,2,3,4, b^{i} \neq b^{j}(i, j=1$, $2,3,4, i \neq j)$

(c) $\left\{\left(\{2,3\},\left\{c^{1}, c^{2}\right\}\right),\left(\{1,3\},\left\{c^{3}, c^{4}\right\}\right)\right\}, c^{i}=1,2,3,4, c^{i} \neq c^{j}(i, j=1$, $2,3,4, i \neq j)$.

Without loss of generality, we choose one optimal observer configuration from all the obtained optimal configuration pairs to verify the proposed FDI algorithm, i.e., $\{(\{1,2\},\{1,2\}),(\{2,3\},\{1$, $2\})\}$. This means that we only need two SUIOs to implement robust FDI. Particularly, SUIO 1 is designed to be sensitive to the faults occurred in the actuator 1 (the left pump) and the actuator 2 (the right pump), and $\omega_{1, k}$ and $\omega_{2, k}$ are decoupled actively, while $\omega_{3, k}$ and $\omega_{4, k}$ are decoupled passively by using their bounds to guarantee FDI robustness. SUIO 2 is designed to be sensitive to the faults in the actuator 2 (the right pump) and the actuator 3 (the middle pump), and $\omega_{1, k}$ and $\omega_{2, k}$ are decoupled actively, while $\omega_{3, k}$ and $\omega_{4, k}$ are decoupled passively to guarantee FDI robustness.

In order to clearly illustrate the effectiveness of the designed optimal SUIOs, we consider comparing two classical guaranteed robust FDI methods with the optimal SUIO-based method proposed in this paper. The first classical FDI method is based on the invariant set theory. The detailed introduction on the invariant-set based FDI method can be referred to $[23,28,29]$. The second algorithm is implemented based on the process that we do not design the optimal parametric matrices while only use some proper parametric

\begin{tabular}{|c|c|c|c|c|c|}
\hline \multirow{4}{*}{$l_{1}=$} & {$[0.5769$} & 0 & 0.0869 & 0 & \multirow{4}{*}{, } \\
\hline & 0 & 0.1381 & 0 & 0.0838 & \\
\hline & 0 & 0 & 0.2311 & 0 & \\
\hline & 0 & 0 & 0 & 0.4259 & \\
\hline \multirow{4}{*}{$L_{2}=$} & {$[0.0769$} & 0 & 0.0869 & 0 & \multirow{4}{*}{ ar } \\
\hline & 0 & 0.2681 & 0 & 0.0838 & \\
\hline & 0 & 0 & 0.6311 & 0 & \\
\hline & 0 & 0 & 0 & 0.7659 & \\
\hline \multirow{4}{*}{$-3=$} & {$[0.4269$} & 0 & 0.0869 & 0 & \\
\hline & 0 & 0.3181 & 0 & 0.0838 & \\
\hline & 0 & 0 & 0.2811 & 0 & \\
\hline & 0 & 0 & 0 & 0.4859 & \\
\hline
\end{tabular}
matrices guaranteeing the stability of SUIOs to implement FDI. In this case, the designed SUIOs are stable but do not own optimality proposed in this paper. Here, we denote this kind of SUIOs as ordinary SUIOs.
For the invariant set-based guaranteed robust FDI method, the observer gain matrix under the Lunberger observer framework corresponding to the healthy situation is designed as

$L_{0}=\left[\begin{array}{cccc}-0.0231 & 0 & 0.0869 & 0 \\ 0 & 0.3581 & 0 & 0.0838 \\ 0 & 0 & 0.3111 & 0 \\ 0 & 0 & 0 & 0.6159\end{array}\right]$ and the remaining observer gain matrices corresponding to the three kinds of faulty situations are designed as

For the design of two ordinary SUIOs, the parametric matrices of the first ordinary SUIO are given as

$$
\begin{aligned}
H_{0} & =\left[\begin{array}{cccc}
0.5114 & 0.4539 & 0.9861 & 0.5589 \\
0.1455 & 0.1687 & 0.8990 & 0.7742 \\
0.5375 & 0.3759 & 0.6307 & 0.0822 \\
0.8955 & 0.2676 & 0.3103 & 0.7278
\end{array}\right], \\
K_{1} & =\left[\begin{array}{cccc}
-0.9467 & 0.0011 & -0.1939 & 0.1967 \\
0.0009 & -0.9797 & -0.0550 & 0.0558 \\
-0.0035 & -0.0011 & -0.7606 & -0.2124 \\
0.0043 & 0.0014 & -0.2561 & -0.7002
\end{array}\right]
\end{aligned}
$$

and the parametric matrices of the second ordinary SUIO are

$$
\begin{aligned}
H_{0} & =\left[\begin{array}{llll}
2.7411 & 4.9067 & 1.3606 & 2.4404 \\
3.8355 & 5.0156 & 6.7283 & 4.7874 \\
4.1458 & 2.5468 & 5.3861 & 1.5716 \\
2.1831 & 4.3818 & 5.7705 & 1.1970
\end{array}\right], \\
K_{1} & =\left[\begin{array}{cccc}
-0.8720 & 0.0008 & -0.0450 & -0.0350 \\
1.3959 & -0.9659 & -0.8057 & -0.6255 \\
-0.3497 & -0.0035 & -0.7681 & 0.1567 \\
0.8788 & 0.0089 & -0.5072 & -1.3538
\end{array}\right] .
\end{aligned}
$$

Given $H_{0}$ and $K_{1}$, the values of the remaining parametric matrices of two ordinary SUIOs can be computed based on the unknown inputs decoupling conditions. The configuration of these two ordinary SUIOs is the same with that of the optimal SUIOs 1 and 2. Thus, the configuration of the first ordinary SUIO corresponding to $(\{1,2\},\{1,2\})$ is same with that of the optimal SUIO 1 and the configuration of the second ordinary SUIO corresponding to $(\{2,3\},\{1,2\})$ is same with that of the optimal SUIO 2. The difference of these two groups of SUIOs only lies in whether the designed parametric matrices are optimal or not.

For the optimal SUIO 1, the related parameters are set as $H_{0}=$ $\left[\begin{array}{llll}0.5114 & 0.4539 & 0.9861 & 0.5589 \\ 0.1455 & 0.1687 & 0.8990 & 0.7742 \\ 0.5375 & 0.3759 & 0.6307 & 0.0822 \\ 0.8955 & 0.2676 & 0.3103 & 0.7278\end{array}\right]$ and the approximation precision is given as $\epsilon=10^{-10}$ and $\lambda=1$. By iterating (29), we can find the proper $k^{*}=6$. For the fault in the actuator $1, k_{f}^{*}=9$, and for the fault in actuator $2, k_{f}^{*}=10$. 
Table 2

Each component of interval hulls of residual sets.

\begin{tabular}{llll}
\hline $\mathcal{R}^{\text {inv }}$ & $\mathcal{R}_{f}^{\text {inv }, 1}$ & $\mathcal{R}_{f}^{\text {inv }, 2}$ & $\mathcal{R}_{f}^{\text {inv, }}$ \\
\hline$(-0.023,0.023)$ & $(-0.034,-0.018)$ & $(-0.013,0.013)$ & $(-0.007,0.007)$ \\
$(-0.008,0.008)$ & $(-0.013,0.013)$ & $(-0.034,-0.011)$ & $(-0.009,0.009)$ \\
$(-0.010,0.010)$ & $(-0.011,0.011)$ & $(-0.029,-0.010)$ & $(-0.060,-0.035)$ \\
$(-0.008,0.008)$ & $(-0.098,-0.069)$ & $(-0.007,-0.007)$ & $(-0.0406,-0.021)$ \\
\hline
\end{tabular}
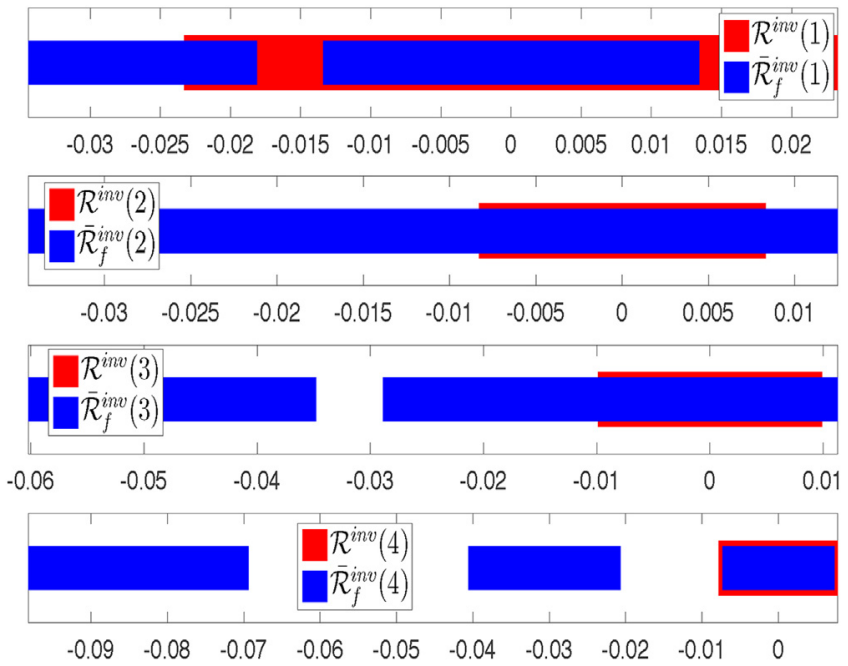

Fig. 3. Results on whether the residual sets are separate for the invariant set-based FDI method.

For the optimal SUIO 2, the related parameters are set as $H_{0}=$ $\left[\begin{array}{llll}2.7411 & 4.9067 & 1.3606 & 2.4404\end{array}\right]$

$\begin{array}{llll}3.8355 & 5.0156 & 6.7283 & 4.7874\end{array}$

$\begin{array}{llll}4.1458 & 2.5468 & 5.3861 & 1.5716\end{array}$

and the approximation pre-

$2.1831 \quad 4.3818 \quad 5.7705 \quad 1.1970$

cision is given as $\epsilon=10^{-10}$ and $\lambda=1$. By iterating (29), we can find the proper $k^{*}=5$. For the fault in the actuator $2, k_{f}^{*}=8$, and for the fault in actuator $3, k_{f}^{*}=8$.

The initial conditions for the two optimal SUIOs 1 and 2 are given as $H_{0}^{e}=\left[\begin{array}{cccc}0.01 & 0 & 0 & 0 \\ 0 & 0.01 & 0 & 0 \\ 0 & 0 & 0.01 & 0 \\ 0 & 0 & 0 & 0.01\end{array}\right]$. We should first verify the setseparation conditions of the three guaranteed FDI methods, which are the premise to achieve guaranteed FDI performance.

Regarding the traditional invariant set-based guaranteed robust FDI method, the intervals of each component of residual sets including one healthy set and three faulty sets are shown in Table $2 . \mathcal{R}^{\text {inv }}$ denotes the residual set when the system operates in healthy situation. $\overline{\mathcal{R}}_{f}^{i n v, i}(i=1,2,3)$ denotes the residual set when the $i$ th actuator is faulty.

For the convenience of presentation, here we compute the union of the three faulty sets on each component and check whether the healthy residual set is separated from the union of faulty sets. That is $\overline{\mathcal{R}}_{f}^{i n v}=\mathcal{R}_{f}^{i n v, 1} \cup \mathcal{R}_{f}^{i n v, 2} \cup \mathcal{R}_{f}^{i n v, 3}$. The results on whether the sets of each component are separated from each other or not are shown in Fig. 3.

Specifically, the four components of the healthy residual set $\mathcal{R}^{\text {inv }}$ and the faulty residual set $\overline{\mathcal{R}}_{f}^{i n v}$ are displayed in the four subplots of Fig. 3. The red region represents the healthy set $\mathcal{R}^{i n v}$, while the blue region represents the faulty set $\overline{\mathcal{R}}_{f}^{i n v}$. Note that, the region height has no meaning and what we concern is whether there exists an overlap on the abscissa axis, i.e., set intersection on the abscissa axis. According to Fig. 3, we can find that the healthy resid-
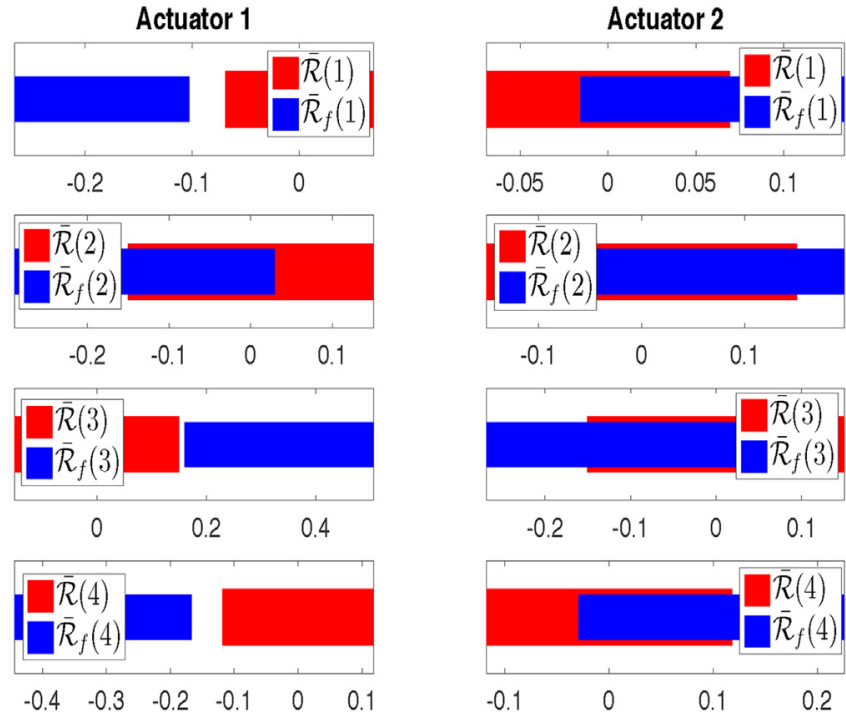

Fig. 4. Results on whether the residual sets are separate for the first ordinary SUIO.
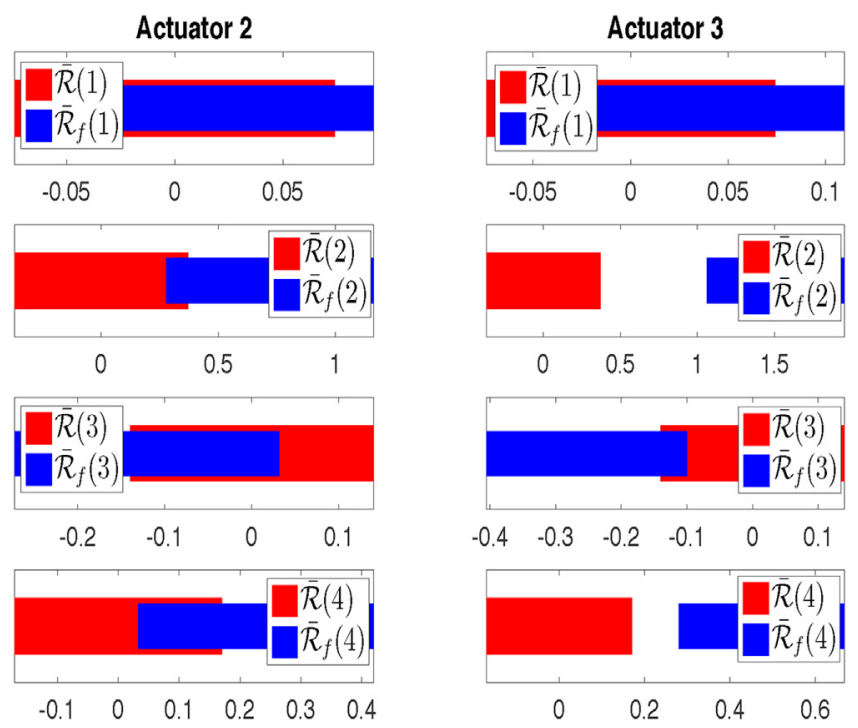

Fig. 5. Results on whether the residual sets are separate for the second ordinary SUIO.

ual set $\mathcal{R}^{i n v}$ is not separated from the faulty union set $\overline{\mathcal{R}}_{f}^{i n v}$, i.e., $\mathcal{R}^{i n v} \cap \overline{\mathcal{R}}_{f}^{i n v} \neq \emptyset$. Since the set-separation conditions are not guaranteed, the invariant set-based method cannot be used for guaranteed robust FDI in this case.

We further check whether the two ordinary SUIOs satisfy the set-separation conditions in Theorem 5 . The results of the first one for the faults in the actuators 1 and 2 are displayed in Fig. 4 and those of the second one for the faults in the actuators 2 and 3 are shown in Fig. 5. We take Fig. 4 as an example for illustration. The four subplots on the left side of Fig. 4 show the results on whether the healthy and faulty residual sets are separate from each other when the actuator 1 is faulty while the actuators 2 and 3 are healthy. The right side corresponds to the situation that the actuator 2 is faulty while the actuators 1 and 3 are healthy.

According to Fig. 4, we can find that for the first ordinary SUIO, the set-separation conditions are only satisfied under the situation that the actuator 1 is faulty while not satisfied when the actuator 2 is faulty. Similar analysis can be done for the second ordinary SUIO. According to Fig. 5, it can be found that the set-separation 

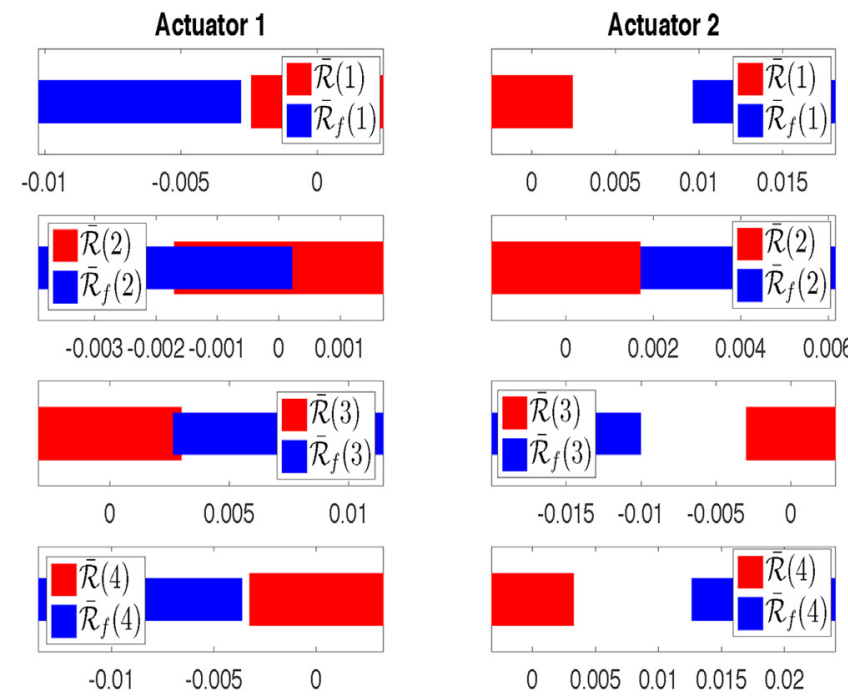

Fig. 6. Set-separation results of the optimal SUIO 1.
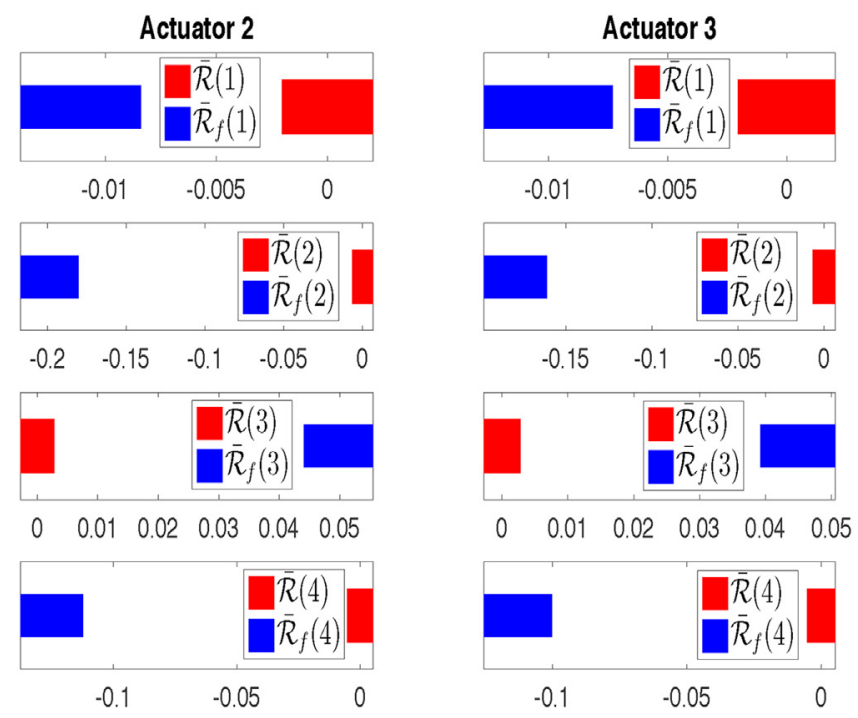

Fig. 7. Set-separation results of the optimal SUIO 2.

conditions of the second ordinary SUIO are only satisfied under the situation that the actuator 3 is faulty while not satisfied when the actuator 2 is faulty. Based on the analysis above, we can conclude that the two ordinary SUIOs without the design of optimal parametric matrices cannot be used for the implementation of guaranteed robust FDI since they do not satisfy the set-separation guaranteed FDI conditions.

Finally, let us verify the set-separation conditions of the optimal SUIOs 1 and 2. The set-separation conditions of the optimal SUIO 1 for the faults in the actuators 1 and 2 are shown in Fig. 6. The set-separation conditions of the optimal SUIO 2 for the faults in the actuator 2 and 3 are shown in Fig. 7.

Taking Fig. 6 as an example to illustrate the set-separation results, the four components of the healthy residual set $\overline{\mathcal{R}}$ and the faulty residual set $\overline{\mathcal{R}}_{f}$ (fault occurred in the actuator 1 ) are displayed in the four subplots on the left side of Fig. 6 . We conclude that the set-separation conditions are satisfied when the fault occurred in the actuator 1, i.e., $\overline{\mathcal{R}} \cap \overline{\mathcal{R}}_{f}=\emptyset$. Similar analysis can be obtained from the right side of Fig. 6 when the actuator 2 is faulty. Therefore, the set-separation conditions of the optimal SUIO 1 for the faults occurred in the actuators 1 and 2 are satisfied. Analogously, the setseparation results of the optimal SUIO 2 for the faults occurred in
Table 3

Fault signature matrix.

\begin{tabular}{lll}
\hline Faults & Optimal SUIOs & \\
\cline { 2 - 3 } & 1 & 2 \\
\hline In Actuator 1 & 1 & 0 \\
In Actuator 2 & 1 & 1 \\
In Actuator 3 & 0 & 1 \\
\hline
\end{tabular}
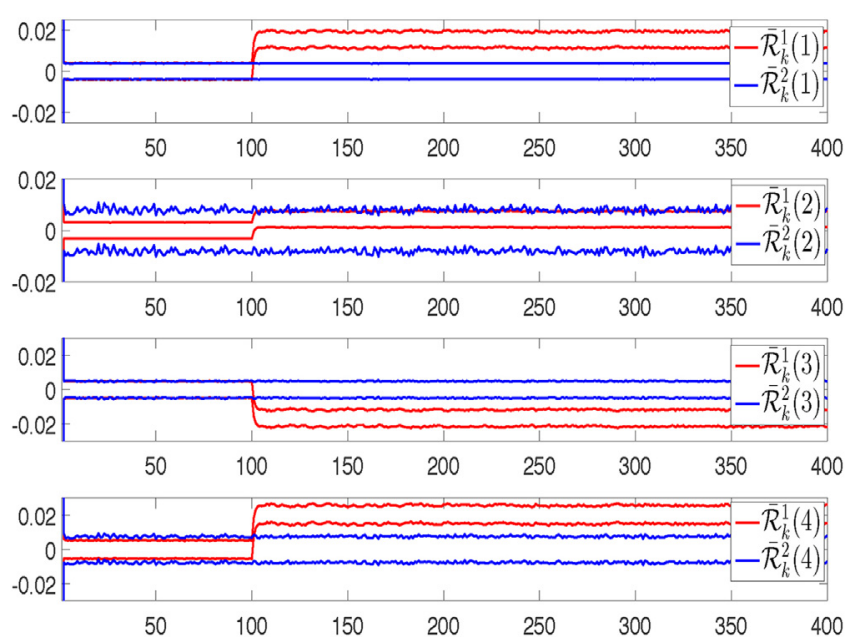

Fig. 8. FDI results of the fault in the actuator 1 .

the actuators 2 and 3 are shown in Figs. 7, and we can find that the set-separation conditions of SUIO 2 (i.e., $\overline{\mathcal{R}} \cap \overline{\mathcal{R}}_{f}=\emptyset$ ) are also satisfied for the faults of the actuators 2 and 3 . In conclusion, compared with the optimal SUIOs 1 and 2, neither the invariant set-based method nor the ordinary SUIOs 1 and 2 satisfy the set separation based guaranteed FDI conditions, which also illustrates the advantages of the proposed optimal SUIOs in the aspect of guaranteeing the set-separation conditions. Moreover, since the set-separation conditions of the invariant set-based method and the ordinary SUIOs are not satisfied, they should be directly excluded and consequently we choose the optimal SUIOs to illustrate the following robust FDI procedure.

According to the set-separation results, we can obtain the fault signature matrix presented in Table 3 . By observing the fault signature matrix, it is known that the faults in the actuators 1,2 and 3 can be distinguished from each other because any two rows in this signature matrix are different from each other, which is guaranteed by the proposed FDI method.

For all the three fault scenarios above, we consider that from $k=0$ to $k=99$, the four tank system operates in healthy situation, while from time instant $k=100$, the faults occur in the corresponding actuators. The FDI results of the fault in the actuator 1 are shown in Fig. 8. The four subplots correspond to the four components of the residual set. The region between two red lines represents the residual set of SUIO 1, while the region between of two blue lines is the residual set of SUIO 2. We can find that from time instant $k=0$ to $k=99$, the origin $\mathbf{0}$ always belongs to the residual sets $\overline{\mathcal{R}}_{k}^{1}$ and $\overline{\mathcal{R}}_{k}^{2}$, i.e., $\mathbf{0} \in \overline{\mathcal{R}}_{k}^{1}$ and $\mathbf{0} \in \overline{\mathcal{R}}_{k}^{2}$. Therefore, we consider that the four-tank system operates in the healthy situation from $k=0$ to $k=99$. From time instant $k=100$, we can find that the origin $\mathbf{0}$ no longer belongs to the residual set $\overline{\mathcal{R}}_{k}^{1}$, i.e., $\mathbf{0} \notin \overline{\mathcal{R}}_{k}^{1}$, which means that the actuator fault has occurred in the system. Moreover, the residual set $\overline{\mathcal{R}}_{k}^{2}$ always contains the origin $\mathbf{0}$, i.e., $\mathbf{0} \in \overline{\mathcal{R}}_{k}^{2}$, during the whole stage of system operation. The FDI results of two optimal SUIOs match the first row of the fault signature matrix of Table 3 , which indicates 

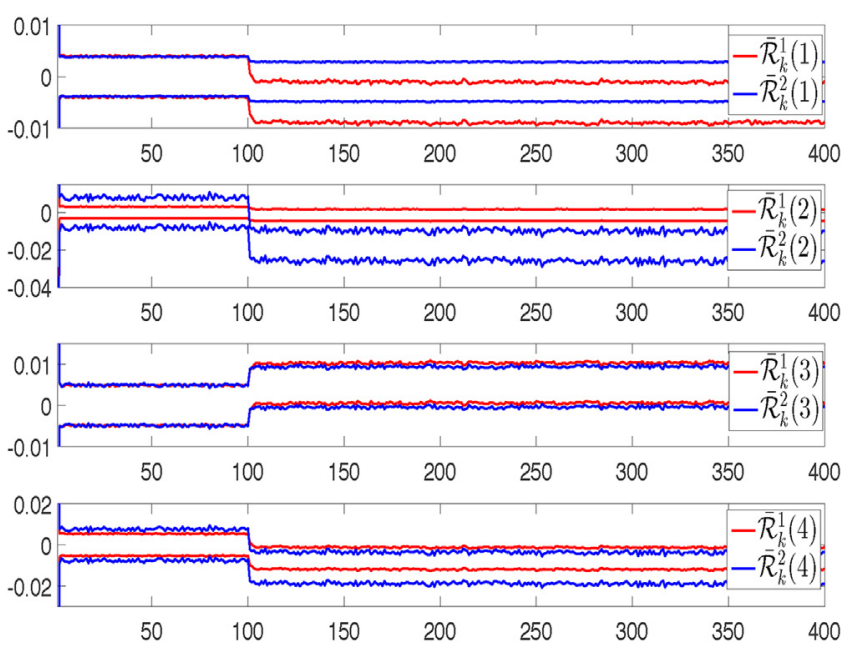

Fig. 9. FDI results of the fault in the actuator 2 .
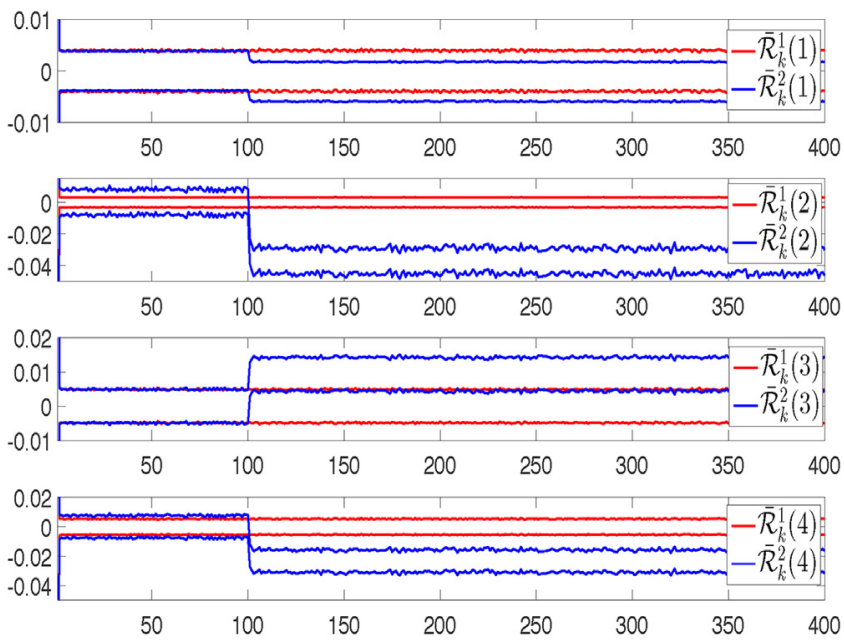

Fig. 10. FDI results of the fault in the actuator 3.

that from $k=100$, the fault occurred in the actuator 1 , while the actuators 2 and 3 are still healthy.

The FDI results of the actuator 2 are shown in Fig. 9. We see that the origin $\mathbf{0}$ is contained in $\overline{\mathcal{R}}_{k}^{1}$ and $\overline{\mathcal{R}}_{k}^{2}$ from $k=0$ to $k=99$, i.e., $\mathbf{0} \in \overline{\mathcal{R}}_{k}^{1}$ and $\mathbf{0} \in \bar{R}_{k}^{2}$, which indicates that the system is healthy. However, from $k=100$, neither $\bar{R}_{k}^{1}$ nor $\overline{\mathcal{R}}_{k}^{2}$ contain the origin $\mathbf{0}$, i.e., $\mathbf{0} \notin \bar{R}_{k}^{1}$ and $\mathbf{0} \notin \overline{\mathcal{R}}_{k}^{2}$, which means that the system operates in the faulty situation. The FDI results of two optimal SUIOs match the second row of the fault signature matrix in Table 3 from $k=100$. Therefore, the fault occurred in the actuator 2, while the actuators 1 and 3 are still healthy.

The FDI results of actuator 3 are shown in Fig. 10. Similar analysis as shown in the previous cases can be done. The four-tank system operates in healthy situation from $k=0$ to $k=99$ for $\mathbf{0} \in \overline{\mathcal{R}}_{k}^{1}$ and $\mathbf{0} \in \overline{\mathcal{R}}_{k}^{2}$, while the fault is detected at $k=100$ because the origin $\mathbf{0}$ no longer belongs to the residual set $\overline{\mathcal{R}}_{k}^{2}$, i.e., $\mathbf{0} \notin \overline{\mathcal{R}}_{100}^{2}$. However, the condition $\mathbf{0} \in \overline{\mathcal{R}}_{k}^{1}$ from $k=100$ is always satisfied, which matches the third row of the fault signature matrix in Table 3. It means that the actuator 3 is faulty, while the actuators 1 and 2 are still healthy.

\section{Conclusions}

This paper has proposed an invariant set-based optimal guaranteed robust FDI method, whose optimality is achieved by considering two different layers. The first layer aims to design the optimal FDI-oriented SUIOs. Moreover, in order to obtain FD guarantees, an invariant set separation-based FD condition is further established for the proposed optimal SUIOs. The second layer consists in sufficiently exploiting potentialities of the FDI scheme using a bank of observers. By making full use of the partitionings of control and unknown inputs for the design of optimal SUIOs, a method to obtain an optimal observer configuration is proposed, which allows the design of a bank of optimal FDI-oriented SUIOs based on this optimal observer configuration for robust FDI. As a result, the proposed FDI method can reduce the effects of unknown inputs on the FDI conservatism and simultaneously obtain FDI guarantees. In the future, we will extend the proposed approach to consider parametric uncertainties and LPV systems.

\section{Acknowledgment}

This work was partially supported by the National Natural Science Foundation of China (No. U1813216), the Science and Technology Research Foundation of Shenzhen (No. JCYJ20170412171459177 and No. JCYJ20170817152701660), and the Spanish State Research Agency (AEI) and the European Regional Development Fund (ERFD) through the project DEOCS (ref. DPI2016-76493-C3-3-R).

\section{References}

[1] M. Blanke, M. Kinnaert, J. Lunze, M. Staroswiecki, Diagnosis and Fault-Tolerant Control, Springer-Verlag, Berlin, Germany, 2006.

[2] Y. Zhang, J. Jiang, Bibliographical review on reconfigurable fault-tolerant control systems, Annu. Rev. Control 32 (2) (2008) 229-252.

[3] F. Xu, V. Puig, C. Ocampo-Martinez, S. Olaru, F. Stoican, Set-theoretic methods in robust detection and isolation of sensor faults, Int. J. Syst. Sci. 46 (13) (2014) 2317-2334.

[4] A. Cristofaro, M.M. Polycarpou, T.A. Johansen, Fault diagnosis and fault-tolerant control allocation for a class of nonlinear systems with redundant inputs, 54th IEEE Conference on Decision and Control (CDC) (2015) 5117-5123.

[5] J. Zhang, A.K. Swain, S.K. Nguang, Detection and isolation of incipient sensor faults for a class of uncertain non-linear systems, IET Control Theory Appl. 6 (12) (2012) 1870-1880.

[6] S. Dey, P. Pisu, B. Ayalew, A comparative study of three fault diagnosis schemes for wind turbines, IEEE Trans. Control Syst. Technol. 23 (5) (2015) 1853-1868.

[7] J. Chen, R. Patton, Robust Model-Based Fault Diagnosis for Dynamic Systems, Kluwer Academic Publishers, 1999

[8] W. Chen, W.T. Chen, M. Saif, M.F. Li, H. Wu, Simultaneous fault isolation and estimation of lithium-ion batteries via synthesized design of Luenberger and learning observers, IEEE Trans. Control Syst. Technol. 22 (1) (2014) 290-298.

[9] F. Caccavale, A. Marino, G. Muscio, F. Pierri, Discrete-time framework for fault diagnosis in robotic manipulators, IEEE Trans. Control Syst. Technol. 21 (5) (2013) 1858-1873

[10] Y. Wang, V. Puig, F. Xu, G. Cembrano, Zonotopic unknown input observer of discrete-time descriptor systems for state estimation and robust fault detection, in: 10th IFAC Symposium on Fault Detection, Supervision and Safety for Technical Processes, Warsaw, Poland, 2018

[11] J. Chen, R. Patton, H. Zhang, Design of unknown input observers and robust fault detection filters, Int. J. Control 63 (1) (1996) 85-105.

[12] S. Hui, S.H. Żak, Observer design for systems with unknown inputs, Int. J. Appl Math. Comput. Sci. 15 (4) (2005) 431-446.

[13] P. Kudva, N. Viswanadham, A. Ramakrishna, Observers for linear systems with unknown inputs, IEEE Trans. Automat. Control 25 (1) (1980).

[14] T. Park, Designing fault detection observers for linear systems with mismatched unknown inputs, J. Process Control 23 (2013) 1016-1022.

[15] Z. Gao, X. Liu, M.Z.Q. Chen, Unknown input observer-based robust fault estimation for systems corrupted by partially decoupled disturbances, IEEE Trans. Ind. Electron. 63 (4) (2015) 2537-2547.

[16] D. Ichalal, S. Mammar, On unknown input observers for LPV systems, IEEE Trans. Ind. Electron. 62 (9) (2015) 5870-5880.

[17] F. Xu, J. Tan, X. Wang, V. Puig, B. Liang, B. Yuan, A novel design of unknown input observers using set-theoretic methods for robust fault detection, in: Proceedings of the 2016 American Control Conference, Boston, USA, 2016.

[18] B. Charandabi, H.J. Marquez, A novel approach to unknown input filter design for discrete-time linear systems, Automatica 50 (2014) 2835-2839.

[19] G. Zheng, F. Bejarano, W. Perruquetti, J. Richard, Unknown input observer for linear time-delay systems, Automatica 61 (2015) 35-43.

[20] S. Varrier, D. Koenig, J. Martinez, Robust fault detection for uncertain unknown inputs LPV system, Control Eng. Pract. 22 (2014) 125-134. 
[21] F. Xu, J. Tan, X. Wang, V. Puig, B. Liang, B. Yuan, H. Liu, Generalized set-theoretic unknown input observer for LPV systems with application to state estimation and robust fault estimation, Int. J. Robust Nonlinear Control 27 (17) (2017) 3812-3832.

[22] V. Puig, J. Quevedo, T. Escobet, A. Stancu, Passive robust fault detection using linear interval observers, in: IFAC Safe Process, Washington, USA, 2003.

[23] M. Seron, J.D. Dona, S. Olaru, Fault tolerant control allowing sensor healthy-to-faulty and faulty-to-healthy transitions, IEEE Trans. Automat. Control 57 (7) (2012) 1657-1669.

[24] J. Blesa, V. Puig, J. Romera, J. Saludes, Fault diagnosis of wind turbines using a set-membership approach, in: Proceedings of the 18th IFAC World Congress, Milano, Italy, 2011.

[25] G. Franzé, F. Tedesco, D. Famularo, Actuator fault tolerant control: a receding horizon set-theoretic approach, IEEE Trans. Automat. Control 60 (8) (2015) 2225-2230.

[26] Y. Wang, M. Zhou, V. Puig, G. Cembrano, Z. Wang, Zonotopic fault detection observer with $\mathcal{H}_{-}$performance, Chinese Control Conference (CCC) (2017) $7230-7235$.

[27] W. Tang, Z. Wang, Y. Shen, Fault detection and isolation for discrete-time descriptor systems based on $\mathcal{H}_{-} / \mathcal{L}_{\infty}$ observer and zonotopic residual evaluation, Int. J. Control (2018), http://dx.doi.org/10.1080/00207179.2018. 1535716.
[28] C. Ocampo-Martinez, J.D. Doná, M. Seron, Actuator fault-tolerant control based on set separation, Int. J. Adapt. Control Signal Process. 24 (12) (2010) 1070-1090.

[29] F. Xu, V. Puig, C. Ocampo-Martinez, F. Stoican, S. Olaru, Actuator-fault detection and isolation based on set-theoretic approaches, J. Process Control 24 (6) (2014) 947-956.

[30] F. Xu, J. Tan, X. Wang, V. Puig, B. Liang, B. Yuan, Mixed active/passive robust fault detection and isolation with set-theoretic unknown input observers, IEEE Trans. Autom. Sci. Eng. 15 (2) (2018) 863-871.

[31] C. Combastel, A state bounding observer based on zonotopes, in: Proceedings of the 2003 European Control Conference, Cambridge, UK, 2003.

[32] T. Alamo, J. Bravo, E. Camacho, Guaranteed state estimation by zonotopes, Automatica 41 (6) (2005) 1035-1043.

[33] S. Olaru, J.D. Doná, M. Seron, F. Stoican, Positive invariant sets for fault tolerant multisensor control schemes, Int. J. Control 83 (12) (2010) 2622-2640.

[34] E. Kofman, H. Haimovich, M. Seron, A systematic method to obtain ultimate bounds for perturbed systems, Int. J. Control 80 (2) (2007) 167-178.

[35] C. Combastel, Zonotopes and kalman observers: gain optimality under distinct uncertainty paradigms and robust convergence, Automatica 55 (2015) 265-273.

[36] D. Limon, M. Pereira, D.M. de la Pena, T. Alamo, J. Grosso, Single-layer economic model predictive control for periodic operation, J. Process Control 24 (8) (2014) 1207-1224. 